\title{
Adhesion between rubber and glass in dry and lubricated condition
}

Cite as: J. Chem. Phys. 148, 234702 (2018); https://doi.org/10.1063/1.5025605

Submitted: 11 February 2018 . Accepted: 30 May 2018 . Published Online: 21 June 2018

L. Dorogin (D, A. Tiwari, C. Rotella, P. Mangiagalli, and B. N. J. Persson

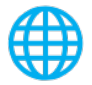

\section{ARTICLES YOU MAY BE INTERESTED IN}

Rubber friction: The contribution from the area of real contact

The Journal of Chemical Physics 148, 224701 (2018); https://doi.org/10.1063/1.5037136

The dependency of adhesion and friction on electrostatic attraction

The Journal of Chemical Physics 148, 144701 (2018); https://doi.org/10.1063/1.5024038

Theory of rubber friction and contact mechanics

The Journal of Chemical Physics 115, 3840 (2001); https://doi.org/10.1063/1.1388626

PHYSICS TODAY

WHITEPAPERS
ADVANCED LIGHT CURE ADHESIVES

Take a closer look at what these

environmentally friendly adhesive

systems can do
READ NOW

PRESENTED BY Q MASTERBOND: 


\title{
Adhesion between rubber and glass in dry and lubricated condition
}

\author{
L. Dorogin,,$^{1,2,3}$ A. Tiwari, ${ }^{2,4}$ C. Rotella, ${ }^{5}$ P. Mangiagalli, ${ }^{5}$ and B. N. J. Persson ${ }^{2, a)}$ \\ ${ }^{1}$ Leibniz Institute for Polymer Research Dresden, P.O. Box 120 411, D-01005 Dresden, Germany \\ ${ }^{2}$ PGI-1, FZ Jülich, Jülich, Germany \\ ${ }^{3}$ ITMO University, Kronverskiy pr. 49, 197101 Saint-Petersburg, Russia \\ ${ }^{4}$ Department of Mechanical and Industrial Engineering (MTP), Norwegian University of Science and Technology, \\ Richard Birkelandsvei 2B, N-7491 Trondheim, Norway \\ ${ }^{5}$ Sanofi, 13, quai Jules Guesde, BP 14, 94403 Vitry sur Seine Cedex, France
}

(Received 11 February 2018; accepted 30 May 2018; published online 21 June 2018)

\begin{abstract}
We study the adhesion between differently processed glass and filled bromobutyl rubber in dry conditions, in water, and in silicone oil. The boundary line between contact and non-contact in adhesion experiments can be considered as a mode I crack, and we show that viscoelastic energy dissipation, close to the opening (or closing) crack tip and surface roughness, strongly affects the work of adhesion. We observe strong adhesion hysteresis and, in contrast to the Johnson-Kendall-Roberts theory prediction for elastic solids, this results in a pull-off force (and work of adhesion) which depends on the loading force and contact time. In particular, for the system immersed in water and silicone oil, we register very weak adhesive bonding. For glass ball with baked-on silicone oil, the pull-off force is nearly independent of the contact time, but this is not observed for the unprocessed glass surface. Published by AIP Publishing. https://doi.org/10.1063/1.5025605
\end{abstract}

\section{INTRODUCTION}

In our everyday practice, most of the contacts manifest rather weak adhesion. ${ }^{1,2}$ However, sometimes adhesion becomes an issue. For instance, clean smooth glass surfaces can exhibit strong adhesion against (smooth) rubber surfaces. Thus, in applications involving rubber in contact with glass, it is often necessary to "passivate" the glass surface, e.g., by siliconisation. One such example is syringes where a rubber stopper must be able to slide with low stable friction against a barrel made from glass. ${ }^{3,4}$ The rubber-glass interface is usually lubricated with silicone oil. However, during a long period of stationary contact, the silicone oil is squeezed out from the rubber-glass asperity contact regions, resulting in a high break loose friction force. In addition, when the syringe is filled with water (with additives), the silicone oil film may be unstable and break up into small droplets (spherical cups). In this case, the rubber stopper can be exposed to bare glass in some contact areas, resulting in a high sliding friction. This can be avoided if silicone oil molecules are chemically attached to the glass surface, which is possible using heat treatment of the (silicone oil covered) glass barrel.

The siliconisation of the syringe barrel is an extremely important aspect of the production of sterile, prefillable glass syringes. Both inadequate and excessive siliconisation can cause problems in this connection. ${ }^{5}$ One option for minimizing the amount of free silicone oil in a syringe is the thermal fixation of the silicone oil on the glass surface in a process called baked-on siliconisation. Usually the silicone oil is applied as an emulsion and then annealed at the temperature $\approx 300{ }^{\circ} \mathrm{C}$.

a)URL: www.MultiscaleConsulting.com.
This creates a permanent hydrophobic anti-friction coating due to the formation of stable covalent bonds between the glass surface and the silicone oil.

The tribology of the contact between glass and rubber has been studied in the pioneering work of Roberts ${ }^{6}$ and of McClune and Briscoe. ${ }^{7}$ Here, smooth rubber surfaces were squeezed against glass in a fluid while being optically observed. It was shown that the flexible rubber resulted in entrapment of liquid by elastic deformation. The removal of fluid (water) between rubber and glass surfaces depends on the wetting properties of the interface, as shown by Roberts and Tabor in Refs. 8 and 9. In the work of Koenen et al., ${ }^{10,11}$ the rubber-glass wiping problem was studied experimentally. They showed that the stick-slip motion and related squeal noise occurred in a narrow range of sliding speeds and loads, which is complicated by capillary adhesion effects that can induce higher friction than in the dry environment.

In this paper, we study the adhesion between "clean" and siliconised glass balls (radius $R$ ) and a flat rubber surface. The relation between adhesion and sliding friction is not trivial, but it is clear that some phenomena such as fluid squeezeout and interfacial aging of the contact should manifest itself in both the adhesion and friction. Thus, the increase in the break loose friction force with the time of stationary contact depends on fluid squeeze-out, dewetting, and bond-formation between the two solids, all of which also influence the adhesive interaction between the solids which can be probed in pull-off experiments. The nature of the break loose friction has been studied in Refs. 12-14 for glass surfaces covered by silicone oil but not for surfaces with baked-on silicone oil.

The contact between a spherical ball and a flat constitutes the simplest and most well-defined contact mechanics problem. For elastically soft solids, and with large radius 
$R$, the Johnson-Kendall-Roberts (JKR) $)^{15-18}$ theory is valid. This theory predicts the pull-off force $F_{\mathrm{c}}=(3 \pi / 2) w R$, where $w=w_{\text {open }}(v)$ is the work of adhesion or, more accurately, the energy (per unit surface area) to propagate an opening crack at the speed $v$ (where $v$ is the crack tip speed at the point of the pull-off instability). In the adiabatic limit (i.e., infinitesimally low pull-off velocity), $w$ is equal to the change in the interfacial free energy $\Delta \gamma=\gamma_{1}+\gamma_{2}-\gamma_{12}$ (where $\gamma_{1}, \gamma_{2}$, and $\gamma_{12}$ are the solid-vapor interfacial energies of solids 1 and 2, and the interfacial energy of the contact between solids 1 and 2 , respectively).

Adhesion mechanics of real objects is a complex phenomenon and is only partly understood, ${ }^{19-28}$ especially for soft materials with viscoelastic and non-linear properties..$^{29,30}$ The work of adhesion during pull-off is strongly influenced by two competing effects: non-adiabatic effects, in particular the viscoelastic energy dissipation in the vicinity of the opening crack tip $^{31,32}$ (which may strongly increase the work of adhesion) and the surface roughness which usually reduces the work of adhesion. ${ }^{33,34}$ We note that the boundary line between contact and non-contact in JKR adhesion experiments can be considered as a mode I crack even if the material on the two sides are different (see, e.g., Ref. 35). The non-adiabatic effects also often result in an adhesion force which is much smaller during approach than during pull-off, an effect referred to as adhesion hysteresis. The effect of surface roughness is considered separately in Refs. 33 and 36-41.

In the present study, we consider the adhesion between bromobutyl rubber and smooth glass surfaces. We consider both dry condition, and in water and in silicone oil. In Sec. II and Appendixes A-C, we present the material properties, namely, the viscoelastic modulus of the rubber, viscoelastic stress and strain relaxation, contact angles and surface energies, and the surface roughness power spectrum of the rubber surface. In Sec. III, we present the results of contact mechanics calculations which form the basis for the analysis of the experimental adhesion data presented in Sec. VI. Section IV discusses viscoelastic crack propagation at the rubber-glass interface. In Sec. V, we present the optical pictures of the contact between a smooth glass surface and the bromobutyl rubber, which illustrates some important aspects of contact hysteresis. In Sec. VI, we consider adhesion between the rubber and glass balls in the dry state, in water, and in silicone oil. Section VII contains the summary and conclusion. Appendixes A-J present details related to material properties and some calculations.

\section{MATERIAL PROPERTIES AND SURFACE TOPOGRAPHY}

\section{A. Viscoelastic modulus}

The viscoelastic properties of rubber are needed for contact mechanics or adhesion calculations and for calculating the velocity dependency of the adhesive crack propagation, e.g., during pull-off. For adhesion study, it is necessary to have information about the complex elastic modulus over a rather large frequency range, as well as at different strain values sometimes (on rough surfaces) including very large strain of order $100 \%$. A standard way of measuring the viscoelastic modulus is to oscillatory deform the rubber sample with a constant strain or stress amplitude. This is done at different frequencies and then repeated at different temperatures. The results measured at different temperatures can be time-temperature shifted to form a master curve at a chosen reference temperature, covering a broad range of frequencies. In Appendix A, we summarize the results obtained for the filled bromobutyl rubber compound used in the adhesion studies.

\section{B. Viscoelastic stress and strain relaxation}

We have performed stress and strain relaxation experiments for the bromobutyl rubber compound. In Fig. 1, we show the time dependency of the relaxation modulus $E(t)$ in units of the relaxation modulus at time $t_{0}=10 \mathrm{~s}$. The green curve is the stress relaxation modulus $E(t)=\sigma(t) / \epsilon_{0}$, where $\epsilon_{0}=0.1$ is the strain imposed at time $t=0$ and $\sigma(t)$ is the resulting stress at time $t$. The solid red curve is the strain relaxation modulus $E(t)$ $=\sigma_{0} / \epsilon(t)$, where $\sigma_{0}=0.4 \mathrm{MPa}$ is the stress imposed at time $t=0$ and $\epsilon(t)$ is the resulting strain at time $t$. The red dashed curve is the strain relaxation modulus calculated from the lowstrain $(0.04 \%)$ master curve $E(\omega)$ shown in Fig. 27. The stress relaxation modulus obtained from the low-strain master curve is nearly the same as the strain relaxation modulus (the dashed red curve) and is not shown in the figure.

Note that the strain relaxation modulus obtained from the small-strain measurement (strain amplitude $0.04 \%$ ) initially decays slower with time than the one obtained for large strain (of order $\sim 10 \%$ ), but for large time the opposite behaviors are observed. The drop in $E(t)$ from the large strain measurements (solid lines) in the time interval of interest below (from $\sim 10^{2}$ $\mathrm{s}$ to $\sim 10^{5} \mathrm{~s}$ ) is about $\approx 25 \%$. The implication of this result for the time dependency of the contact area and the pull-off force will be discussed below.

\section{Contact angles and surface energies}

In order to study the adhesion in water and silicone oil, we need to know the interfacial energies between the rubber and glass and the two fluids, which can be estimated from contact

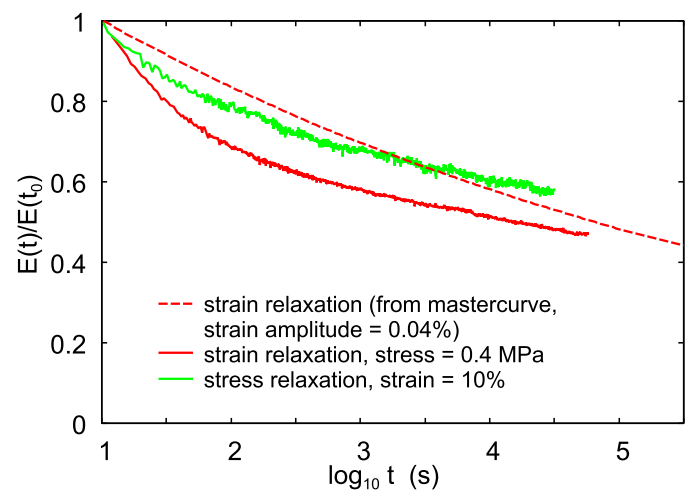

FIG. 1. The time dependency of the relaxation modulus $E(t)$ of the rubber in units of the relaxation modulus at time $t_{0}=10 \mathrm{~s}$. The green curve is the stress relaxation modulus $E(t)=\sigma(t) / \epsilon_{0}$, where $\epsilon_{0}=0.1$ is the strain imposed at time $t=0$ and $\sigma(t)$ is the resulting stress at time $t$. The solid red curve is the strain relaxation modulus $E(t)=\sigma_{0} / \epsilon(t)$, where $\sigma_{0}=0.4 \mathrm{MPa}$ is the stress imposed at time $t=0$ and $\epsilon(t)$ is the resulting strain at time $t$. The red dashed curve is the strain relaxation modulus calculated from the low-strain $(0.04 \%)$ master curve $E(\omega)$ shown in Fig. 27. 
angle measurements. We have measured the contact angles for water and silicone oil on the glass ball surface and on the surface of the bromobutyl rubber. Using this information, we have estimated the adiabatic work of adhesion between the rubber and the glass surface, in the dry state, in water, and in silicone oil. Here we summarize the most important results (see Appendix B for details).

In the dry state for long contact time, the adhesive JKR contact is characterized by the adiabatic work of adhesion (for perfectly smooth surfaces) $\Delta \gamma=\gamma_{1}+\gamma_{2}-\gamma_{12}$. Assuming that only dispersion forces are responsible for the interaction between the glass and the bromobutyl rubber; in Appendix B, we estimate the adiabatic work of adhesion $\Delta \gamma($ dry $) \approx 0.08$ $-0.1 \mathrm{~J} / \mathrm{m}^{2}$. However, the experimental adhesion study presented below indicates that stronger bonds form between the two surfaces and $\Delta \gamma \approx 0.3 \mathrm{~J} / \mathrm{m}^{2}$.

In a liquid, the adiabatic work of adhesion can be calculated using the Young-Dupre equation

$$
\Delta \gamma(\text { wet })=\Delta \gamma(\text { dry })-\gamma\left(\cos \theta_{\mathrm{gl}}+\cos \theta_{\mathrm{rl}}\right),
$$

where $\gamma$ is the liquid surface tension and $\theta_{\mathrm{gl}}$ is the contact angle of the liquid on glass and $\theta_{\mathrm{rl}}$ is the contact angle of liquid on the rubber. Using this equation with $\Delta \gamma($ dry $) \approx 0.3 \mathrm{~J} / \mathrm{m}^{2}$ gives in water $\Delta \gamma($ wet $) \approx 0.2 \mathrm{~J} / \mathrm{m}^{2}$.

In Appendix B, we argue that the interaction potential between a flat glass surface and a flat rubber surface in water takes the form shown in Fig. 2 with a repulsive barrier before the strong attraction due to direct rubber-glass bonds. That is, at separation of order a few nanometers, the interaction is only via dispersion forces and steric repulsion and is repulsive. At short separation, the interaction potential has a local minimum corresponding to direct contact between the rubber and the glass surface. In this state, bonding forces (of unknown nature) stronger than the dispersion forces occur between the surfaces.

When a bromobutyl rubber is squeezed in contact with the glass surface in water, we expect first a rapid squeeze-out (see Sec. III) until contact occurs between the rubber asperities and the glass surface. However, at short contact time, we do not expect any true atomic contact between the rubber

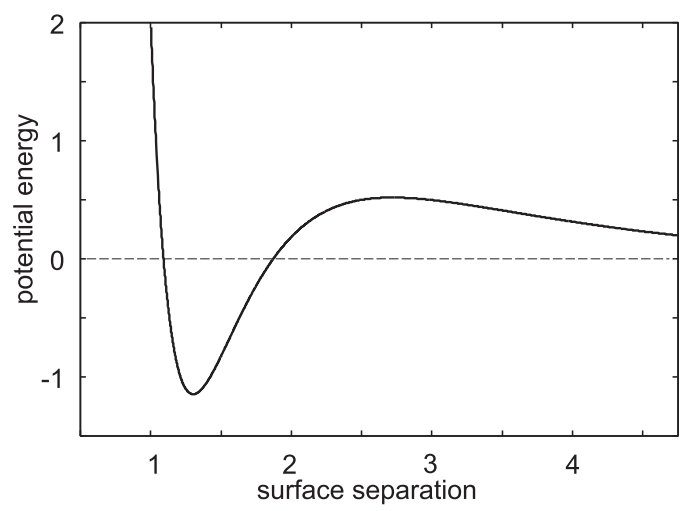

FIG. 2. The interaction potential between a flat glass surface and a flat rubber surface in water as a function of the surface separation. At separation of order a few nanometers, the interaction is only via dispersion forces and steric repulsion and is repulsive. At short separation, the interaction potential has a minimum corresponding to direct contact between the rubber and the glass surface. In this case, bonding forces (of unknown nature) stronger than the dispersion forces occur between the surfaces. Arbitrary units and schematic. and the glass surface, but a water film of nanometer thickness separates the surfaces in the asperity contact regions. However, this state is only metastable and after a long enough time we expect true atomic contact to form between the rubber and the glass surface in the asperity contact regions. This dewetting transition involves the nucleation (by thermal fluctuations) of the nanometer sized contact region followed by the removal of the nanometer water film by a (mainly) surfaceenergy driven squeeze-out process. Thus we expect the contact area between the rubber and the glass surface to increase continuously with increasing contact time, as indeed observed (see Sec. VI).

\section{Rubber surface roughness}

We have studied the rubber surface topography using an optical method and atomic force microscopy (AFM). As shown in Appendix $\mathrm{C}$, the height probability distribution is nearly Gaussian, with the root-mean-square (rms) roughness, when measured over a surface area $1 \mathrm{~mm} \times 1.4 \mathrm{~mm}$, of about $h_{\mathrm{rms}}$ $\approx 2.9 \pm 0.1 \mu \mathrm{m}$ and with the highest point $\sim 13 \mu \mathrm{m}$ above the average plane. In Appendix $\mathrm{C}$, we also give the surface roughness power spectrum which is needed for the adhesion and fluid squeeze-out calculations.

\section{THEORY: CONTACT AREA AND INTERFACIAL SEPARATION}

As a preparation for the analysis of the adhesion data to be presented in Secs. V and VI, here we present some theoretical predictions for the contact between the bromobutyl rubber and smooth glass. We first study adhesion using the theory developed in Refs. 34 and 42, which accounts for the influence of the surface roughness on work of adhesion and the contact area. Next we calculate the time-dependent average interfacial separation, and the area of real contact, during fluid squeezeout between a rubber block and the glass surface. The fluid is assumed to be Newtonian, and we neglect the influence of interfacial energies on the squeeze-out process, i.e., no dewetting transition occurs. The calculations are performed using the Reynolds equations for fluid flow, where the surface roughness enters via flow factors, which are obtained using the Persson contact mechanics theory and the Bruggeman effective medium theory (see Ref. 43).

In the following, we will present results for the work of adhesion and we will use the following notation. The adiabatic work of adhesion for the rubber in contact with the glass surface for perfectly smooth surfaces will be denoted by $\gamma_{0}$ $=\Delta \gamma$. We also refer to this as the interfacial binding energy per unit surface area. For an opening crack, we denote the work of adhesion (for perfectly smooth surfaces) by $\gamma_{\text {open }}(v)$ which depends on the crack tip velocity $v$. Similarly, $\gamma_{\text {close }}(v)$ denotes the work of adhesion for a closing crack. Note that $\gamma_{\text {close }}(v)$ $<\Delta \gamma<\gamma_{\text {open }}(v)$ and that as $v \rightarrow 0$ we have $\gamma_{\text {open }}(v) \rightarrow \Delta \gamma$ and $\gamma_{\text {close }}(v) \rightarrow \Delta \gamma$. For the surfaces with roughness, we denote the adiabatic work of adhesion by $w_{0}$ and the corresponding opening crack and closing crack propagation energies by $w_{\text {open }}(v)$ and $w_{\text {close }}(v)$. Note that $w_{\text {close }}(v)<w_{0}<w_{\text {open }}(v)$ and $w_{\text {open }}(v) \rightarrow w_{0}$ and $w_{\text {close }}(v) \rightarrow w_{0}$ as the crack tip velocity 
$v \rightarrow 0$. In Secs. V and VI, we focus mainly on $w_{\text {open }}(v)$ which we often denote by $w(v)$ or just $w$ for simplicity.

\section{A. Influence of surface roughness on the adhesion}

In this section, we study the influence of surface roughness on the contact between rubber and glass balls in the dry state, and in water and in silicone oil. We assume infinite long contact time so that viscoelastic effects, and other nonadiabatic processes, are unimportant. In this limit, in the dry state, the adhesive contact is characterized by the adiabatic work of adhesion (for perfectly flat surfaces) $\Delta \gamma=\gamma_{1}+\gamma_{2}$ $-\gamma_{12}$. In the case of adhesion in a liquid (here water or silicone oil), we assume that the liquid pressure is everywhere equal to the external pressure so the total load is carried by the area of real contact. In a liquid, the adiabatic work of adhesion can be calculated using the Young-Dupre equation (1).

We will now present calculated results for the adhesive contact between the glass ball and the surface of the bromobutyl rubber. Figure 3(a) shows the normalized area of real contact, $A / A_{0}$ (where $A_{0}$ is the nominal contact area), as a function of the nominal contact pressure (in MPa), without adhesion (the green curve) and with adhesion (red and blue curves). The rubber surface is assumed to have surface roughness with the power spectrum shown in Fig. 32 (the dashed line) and the Young's modulus $E=2.5 \mathrm{MPa}$ and Poisson ratio $v=0.5$. The work of adhesion used for obtaining the red curve is $\Delta \gamma=0.1 \mathrm{~J} / \mathrm{m}^{2}$, and that for the blue curve is $\Delta \gamma=0.025$ $\mathrm{J} / \mathrm{m}^{2}$. These values for the work of adhesion are smaller than
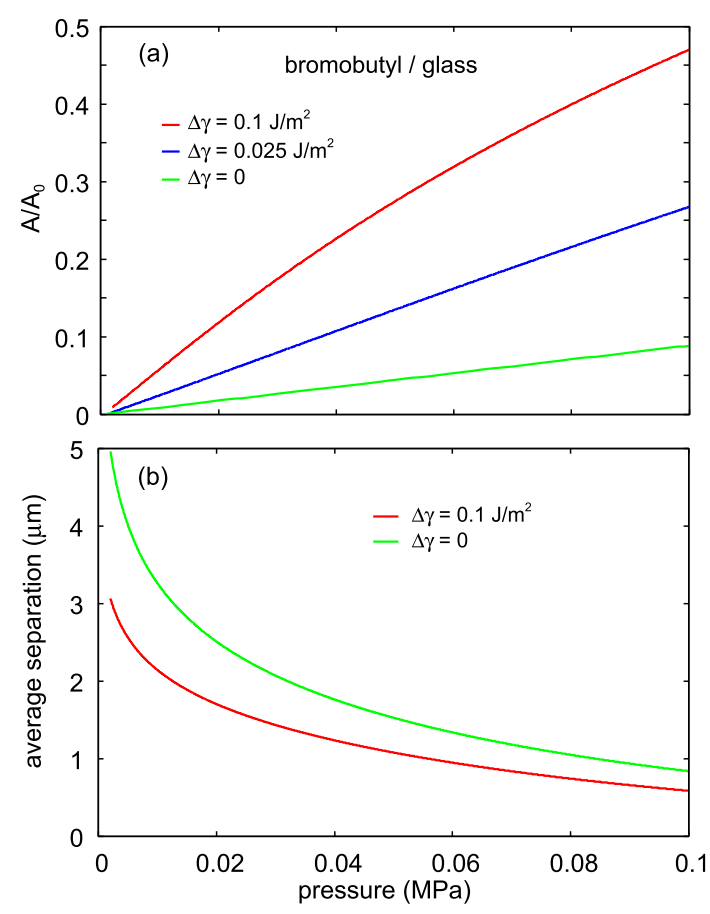

FIG. 3. The (a) normalized area of real contact, $A / A_{0}$ (where $A_{0}$ is the nominal contact area) and (b) the average surface separation, as a function of the nominal contact pressure (in MPa), without adhesion (the green curve) and with adhesion (red and blue curves) found from Persson's theory. The rubber surface is assumed to have surface roughness with the power spectrum shown in Fig. 32 (the dashed line) and the Young's modulus $E=2.5 \mathrm{MPa}$ and the Poisson ratio $v=0.5$. The work of adhesion used for obtaining the red curve is $\Delta \gamma=0.1 \mathrm{~J} / \mathrm{m}^{2}$, and that for the blue curve is $\Delta \gamma=0.025 \mathrm{~J} / \mathrm{m}^{2}$. the adiabatic value (which may be $\approx 0.3 \mathrm{~J} / \mathrm{m}^{2}$ ), but during contact formation the work of adhesion is reduced. Results for $\Delta \gamma=0.3 \mathrm{~J} / \mathrm{m}^{2}$ will be shown below.

Figure 3(b) shows the average surface separation as a function of the nominal contact pressure with adhesion $(\Delta \gamma$ $=0.1 \mathrm{~J} / \mathrm{m}^{2}$, the red curve) and without adhesion (the green curve).

In contact mechanics for the rough surface with roughness over many decades in length scales, the concept of magnification is very important. ${ }^{42}$ When we study the interface at the magnification $\zeta$, we only observe the surface roughness with wavenumber $q<\zeta q_{0}$, where $q_{0}$ is a reference wavenumber, here chosen as $q_{0}=10^{4} \mathrm{~m}^{-1}$, corresponding to the reference length $\lambda_{0}=2 \pi / q_{0} \approx 0.6 \mathrm{~mm}$. Physical quantities, such as the contact area, depend on the magnification used when studying the contact. For example, if $\zeta$ is small (of order 1) we do not observe any surface roughness and it appears as if the contact is complete, i.e., $A / A_{0} \approx 1$. Figure 4 shows the interfacial binding energy (or work of adhesion) (a) and the normalized area of real contact (b), as a function of the logarithm of the magnification $\zeta$. Note that the work of adhesion $w_{0}$ vanishes when $\Delta \gamma=0.1 \mathrm{~J} / \mathrm{m}^{2}$. In fact, the surface roughness results in a vanishing work of adhesion for $\Delta \gamma<0.25 \mathrm{~J} / \mathrm{m}^{2}$ (not shown).

Figure 5 shows the (a) normalized area of real contact and (b) the work of adhesion, as a function of the logarithm of the magnification $\zeta$ when $\Delta \gamma=0.3 \mathrm{~J} / \mathrm{m}^{2}$. Note that $w_{0}(\zeta=1)$ $\approx 0.03 \mathrm{~J} / \mathrm{m}^{2}$, which implies that there is a non-vanishing
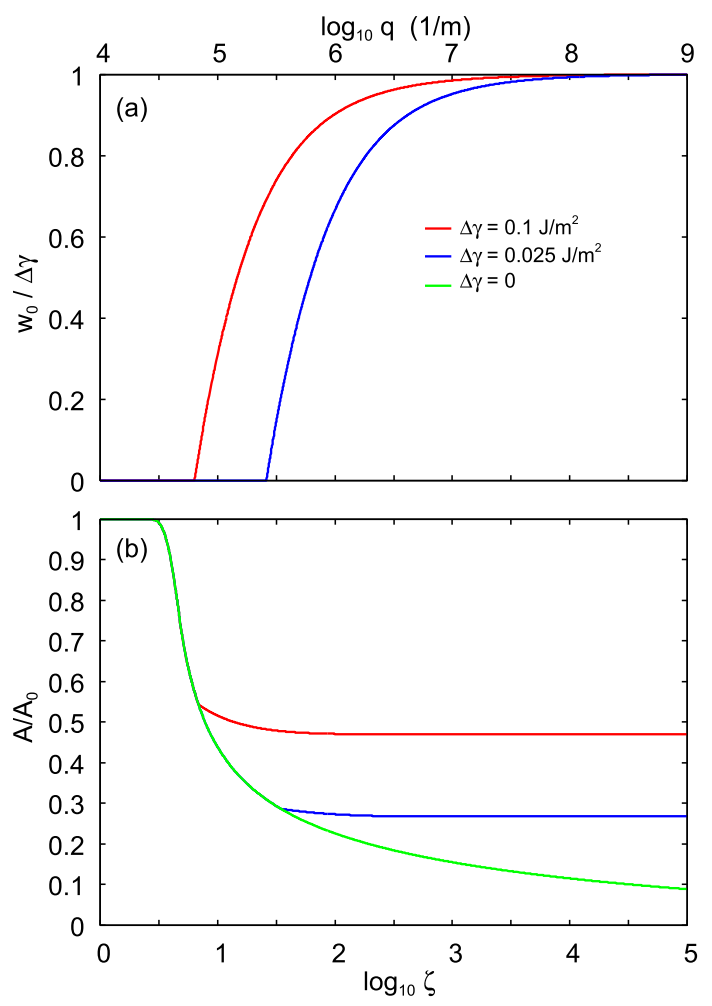

FIG. 4. The (a) interfacial binding energy (or work of adhesion) and (b) the normalized area of real contact, as a function of the logarithm of the magnification $\zeta$ found from Persson's theory. Note that for both $\Delta \gamma=0.1 \mathrm{~J} / \mathrm{m}^{2}$ (the red curve) and $\Delta \gamma=0.025 \mathrm{~J} / \mathrm{m}^{2}$ (the blue curve) $w_{0}(\zeta=1)=0$ which implies that there is vanishing pull-off force in the adiabatic limit (infinitely small pull-off velocity). For the same parameters as in Fig. 3. 

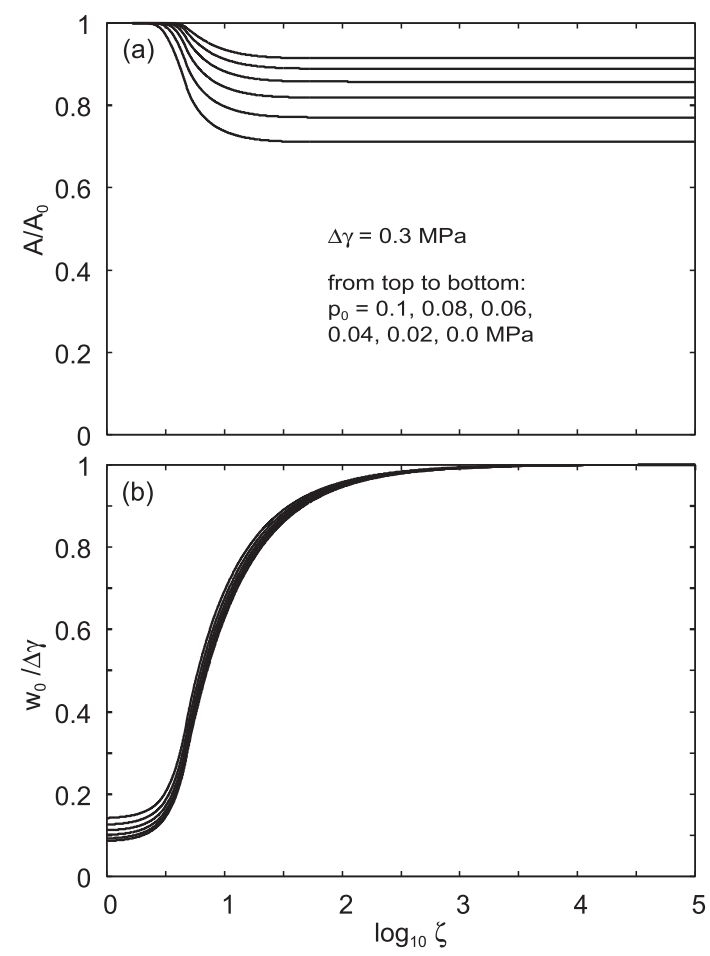

FIG. 5. The (a) normalized area of real contact and (b) the interfacial binding energy (or work of adhesion), as a function of the logarithm of the magnification $\zeta$ when $\Delta \gamma=0.3 \mathrm{~J} / \mathrm{m}^{2}$ found from Persson's theory. Note that $w_{0}$ $(\zeta=1) \approx 0.03 \mathrm{~J} / \mathrm{m}^{2}$, which implies that there is a non-vanishing pull-off force.

pull-off force. Here we note that in reality strong adhesion hysteresis occurs that will be discussed in more detail in Sec. V. Thus, during contact formation for short contact time, the interfacial binding energy nearly vanishes, while it is strongly enhanced during pull-off. Figure 3 shows that if the nominal contact pressure $p_{0} \approx 0.1 \mathrm{MPa}$, for relatively short times where adhesion can be neglected, $A / A_{0} \approx 0.1$. However, after very long time, the interfacial binding energy will approach the adiabatic interfacial binding energy, $\gamma \approx 0.3 \mathrm{~J} / \mathrm{m}^{2}$, and, in this case from Fig. 5 for $p_{0} \approx 0.1 \mathrm{MPa}$, we expect $A / A_{0} \approx 0.9$. Thus we expect the contact area to increase slowly with time from a relative small value, of order $A / A_{0} \approx 0.1$, to a value close to complete contact for very long contact times.

Another very important implication of adhesion hysteresis is the following: during removal of the loading force, the contact area will not drop to zero but will stay nearly unchanged. The reason is that for the opening crack (crack tip velocity $v$ ) the interfacial binding energy $\gamma_{\text {open }}(v) \gg \Delta \gamma$ unless $v$ is extremely small so that the decrease in the contact area with increasing time after removing the load is so slow that it can be neglected on the time scale of practical importance. If the contact area is unchanged, we also expect only a relative small increase in the average interfacial separation when the load is removed. For contact in fluids (water and silicone oil), we also observe (a weak) adhesion and the results presented in Sec. VI indicate that in these cases too the contact area and the average interfacial separation change rather little when the load is reduced from the maximum (where the nominal contact pressure is about $0.1 \mathrm{MPa}$ ) to zero. Optical pictures of the contact in water (see Sec. VI) support this claim.

\section{B. Fluid squeeze-out}

We will show that the viscous forces in silicone oil (and hence also in water) have a negligible influence on the adhesion except in a short time period around detachment. Let us first estimate the typical contact pressures in the applications below. The maximum applied normal forces used below are of order $F=0.1-0.2 \mathrm{~N}$. At these loads, we can neglect the adhesion when estimating the nominal contact pressures. When a spherical ball with radius $R$ is squeezed against a flat surface with the normal force $F$, according to the Hertz theory, the radius of the circular contact region is

$$
r_{0}=\left(\frac{3 F R}{4 E^{*}}\right)^{1 / 3},
$$

where $E^{*}=E /\left(1-v^{2}\right)$. The maximum nominal contact pressure is

$$
p_{0}=\frac{3 F}{2 \pi r_{0}^{2}} .
$$

Note that the contact pressure scale with the loading force as $p_{0} \sim F^{1 / 3}$. Using $E=2.5 \mathrm{MPa}, v=0.5, R=1.5 \mathrm{~cm}$, and $F=0.1 \mathrm{~N}$ gives $r_{0} \approx 0.7 \mathrm{~mm}$, and $p_{0} \approx 0.1 \mathrm{MPa}$.

Consider the squeezing of the glass ball against the rubber surface in silicone oil. The glass ball is assumed to be perfectly smooth, while the rubber surface has surface roughness with a power spectrum shown in Fig. 32. As shown elsewhere, ${ }^{44}$ the separation between the glass ball and the substrate as a function of time can be accurately described by a simpler model where the glass ball is replaced with a circular disc with the same radius $r_{0}$ as the radius of the Hertz contact region when the ball is squeezed against the rubber (with the same normal force) without a liquid. The load acting on the circular disk is the same as that on the glass ball. We will now present results for the interfacial separation and the area of real contact using this mapping of a ball on a disc.

Let us consider a circular disc with radius $r_{0}=0.7 \mathrm{~mm}$ being squeezed against a nominal flat surface in a Newtonian fluid with the viscosity $\eta=0.35$ Pa s, see Fig. 6 showing (a) the logarithm of the interfacial separation and (b) the relative area of real contact as a function of the logarithm of the contact time. The initial separation is $0.1 \mathrm{~mm}$, and the loading force increases linearly with time from 0 to its final value $0.1 \mathrm{~N}$ during the first $0.01 \mathrm{~s}$. We observe that for $p=0.1 \mathrm{MPa}$ (green lines) after $\sim 100 \mathrm{~s}$ the disc (or the ball) has reached its final state where the whole load is carried by the rubber asperity contact regions. Since the loading (and unloading) times in our experiments usually are longer than $\sim 100 \mathrm{~s}$, we conclude that there is very little viscous resistance to the motion of the ball at any stage in the loading and unloading phase, except for a short time interval following the adhesive bond breaking, where the ball would snap-off very fast if there would be no fluid (viscous) damping effect. In fact, in the JKR theory (for elastic solids and neglecting inertia effects), snap-off occurs instantaneously (with infinite high speed), but in a fluid the motion will be damped. In water, the viscous damping is very small (and cannot be resolved in the experiments presented below, where the time resolution is $1 \mathrm{~s}$ ), but in the silicon oil (which has 350 times higher viscosity than water), 

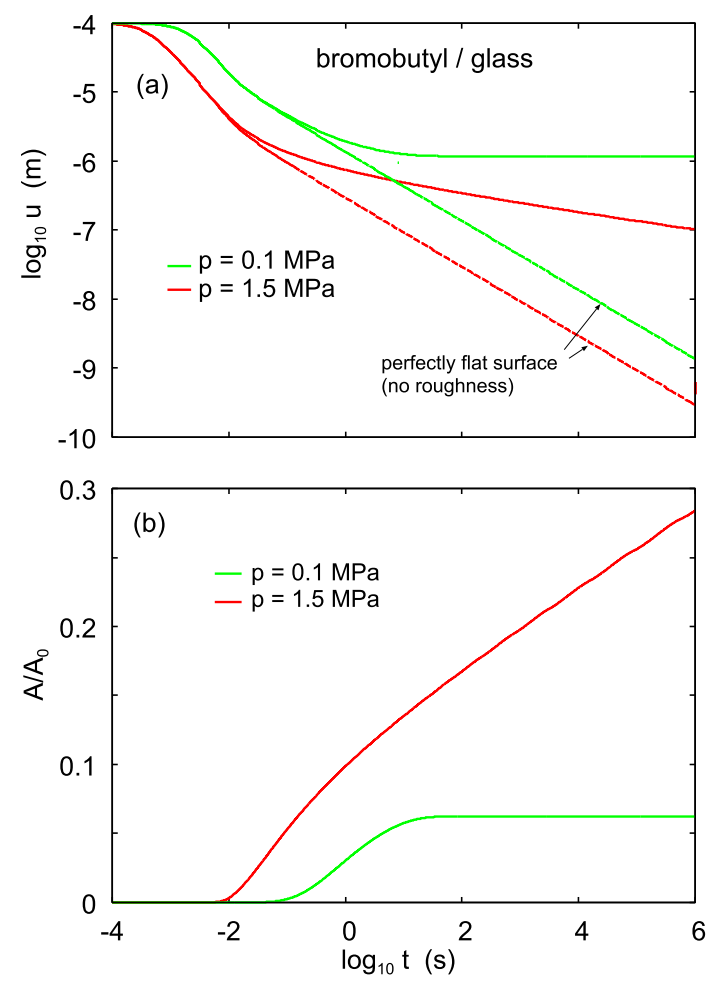

FIG. 6. The (a) logarithm of the interfacial separation and (b) the relative area of real contact, as a function of the logarithm of time, in the model with a circular disk of radius $0.7 \mathrm{~mm}$ squeezed against a nominally flat surface in a Newtonian fluid with the viscosity $\eta=0.35 \mathrm{~Pa}$ s. The substrate surface has the surface roughness power spectrum shown in Fig. 32 (the dashed line), and the Young's elastic modulus $E=2.5 \mathrm{MPa}$ and Poisson ratio $v=0.5$. We show results for the squeezing pressures $p=0.07 \mathrm{MPa}$ (green lines) (as relevant for the experiments presented in this paper) and for a much higher pressure $p=$ 1.5 MPa (red lines), as typical for rubber seals or syringe applications. In (a), the lower red and green lines show the interfacial separation for perfectly flat surfaces (no surface roughness), while the other two lines is with the surface roughness included.

the snap-off extends over $\sim 100 \mathrm{~s}$ in agreement with theory predictions (see Appendixes I and J). Since the squeeze-out time is proportional to the fluid viscosity, for water we expect that it takes less than $1 \mathrm{~s}$ for the disc (or the ball) to reached its final state.

For the higher nominal contact pressure $p=1.5 \mathrm{MPa}$ (red lines), the situation is completely different. Although this case is not of relevance for the experiments presented below, we will discuss this case in some detail as this is the typical contact pressure prevailing in most engineering applications, e.g., for rubber seals or in the contact regions between the ribs of a rubber stopper and the barrel in syringe applications. At the contact pressure $p=1.5 \mathrm{MPa}$, in the absence of the fluid, nearly complete contact occurs between the solids in the nominal contact area, i.e., $A / A_{0} \approx 1$. However, in a fluid, when the relative contact area reaches $A / A_{0} \approx 0.4$, the area of real contact percolates, and no fluid can flow at the interface; this results in trapped islands of pressurized fluid at the interface. Hence, in a fluid, at least in the absence of adhesion (dewetting), when during squeezing the relative contact area approaches $A / A_{0}$ $\approx 0.4$, the fluid flow at the interface (squeeze-out) will slow down. This will result in almost infinitely long squeeze-out times when the external load is large enough to give $A / A_{0}>0.4$ for dry surfaces. This is the origin of the long-time dependency of $u(t)$ and $A(t)$ shown in Fig. 6 for $p=1.5 \mathrm{MPa}$. We note that this effect is of great importance in applications, e.g., to seals or syringes.

\section{VISCOELASTIC CRACK PROPAGATION}

It is well known that the boundary line between contact and non-contact in JKR adhesion experiments can be considered as a mode I crack even if the material on the two sides are different (see, e.g., Ref. 35). For viscoelastic materials, the (opening) crack propagation energy (per unit surface area) can be written as $\gamma_{\text {open }}(v, T)=\Delta \gamma[1+f(v, T)]$, where $\Delta \gamma$ is the crack propagation energy as the crack tip velocity $v \rightarrow 0$. From the measured viscoelastic modulus we can calculate the enhancement factor $[1+f(v, T)]$ to interfacial crack propagation (see Appendix D), which is important for understanding the adhesion data to be presented later.

Using the theory developed in Ref. 31, in Fig. 7 we show the viscoelastic crack propagation factor $[1+f(v, T)]$ as a function of the logarithm of the crack tip velocity $v$. The results are for the bromobutyl rubber compound at $T=20^{\circ} \mathrm{C}$. The red line uses the low-strain $(0.04 \%$ strain $)$ modulus, and the green line uses the large-strain $(\approx 15 \%$ strain) modulus. The vertical dashed line indicates a typical crack tip velocity in the experiments reported on in Sec. VI.

For closing crack propagation, as it is involved in the JKR experiments when the ball is squeezed against the substrate, the crack propagation energy $\gamma_{\text {close }} \approx \Delta \gamma /[1+f(v, T)]$. Hence the viscoelastic energy dissipation can result in a strong adhesion

(a)
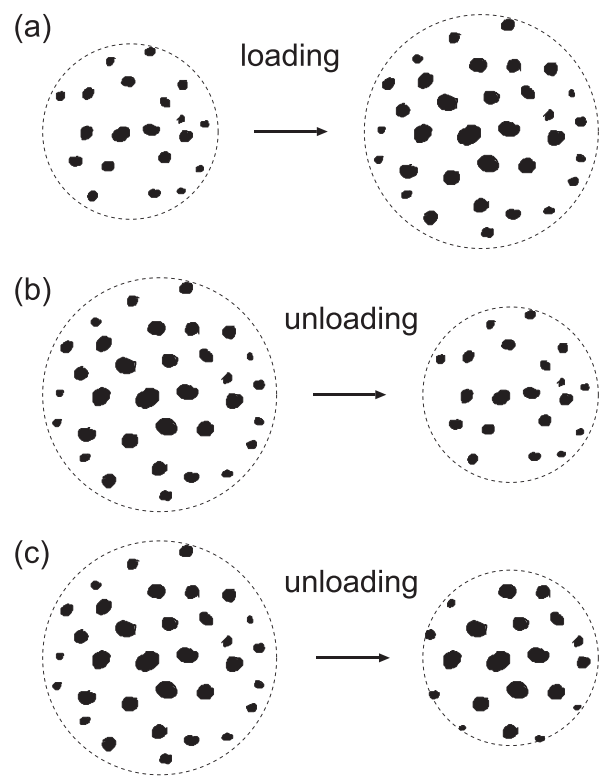

FIG. 7. (a) Schematic picture of the macroscopic contact area during loading, (b) during unloading in the absence of adhesion hysteresis, and (c) when strong adhesion hysteresis occurs so that $\gamma_{\text {open }} \gg \Delta \gamma$. The black regions indicate asperity contact regions. Because of the adhesive interaction, complete contact occurs within the black regions. During pull-off in case (b) the asperity contact regions decrease in size everywhere. During pull-off in case (c) the size of the asperity contact regions remains unchanged (in spite of the reduction in the contact pressure) except close to the macroscopic (apparent) opening crack tip (dashed circle), where the asperity contact regions are broken by the propagation of microscopic opening cracks at each asperity contact region. 
hysteresis, where $\gamma(v, T)$ during contact formation is much smaller than during pull-off.

\section{ADHESION HYSTERESIS}

Let us now include the effect of adhesion hysteresis which we left out of consideration in Section III. We will use the argument first presented in Refs. 45 and 46 to show that the pull-off force is always finite when adhesion hysteresis prevails, assuming that it is big enough.

Assume that at the end of the loading cycle the asperity contact regions appear as shown in Fig. 7(a). In the absence of adhesion hysteresis [Fig. 7(b)], during unloading the asperity contact regions would disappear in a similar way as they were formed. Asperity contact regions even at the center of the macroscopic contact area will decrease in size even at the start of unloading, where the radius $r(t)$ of the macroscopic separation line (dashed lines in Fig. 7) is far from the center of the contact region. However, if the adhesion hysteresis is large enough so that the energy per unit area for the opening crack $\gamma_{\text {open }}(v) \gg \Delta \gamma$, then the asperity contact regions will only start to shrink when they are very close to the macroscopic opening crack (in the crack-tip process zone) [Fig. 7(c)]. In this case, the pull-off force will be non-vanishing, and to a good approximation given by the JKR theory with $w \approx \gamma_{\text {open }}(v) A_{1} / A_{0}$, where $\gamma_{\mathrm{open}}(v)$ is the work of adhesion (for opening crack) obtained from the contact between smooth surfaces and $A_{1} / A_{0}$ is the normalized area of real contact. This is illustrated in Fig. 7(c), where the size of the asperity contact regions outside of the crack-tip process zone remains unchanged (in spite of the reduction in the contact pressure). Very close to the tip of the macroscopic (apparent) opening crack (dashed circle), the asperity contact regions are broken by the propagation of microscopic opening cracks at each asperity contact region.

We now present results for how the adhesion force depends on the applied (maximum) load or normal force. As pointed out above, when strong adhesion hysteresis occurs the work of adhesion during separation will be $w \approx \gamma_{\text {open }}(v) A_{1} / A_{0}$. The relative contact area $A_{1} / A_{0}$ will be close to the relative contact area when the load is maximal. Assuming that the adhesion is weak during approach we expect from the Hertz contact theory that the nominal contact pressure depends on the load as $F_{\mathrm{N}}^{1 / 3}$. If we assume that $A_{1} / A_{0}$ depends linearly on the nominal contact pressure (as it is the case as long as adhesion is not important during approach and $A_{1} / A_{0}$ is smaller than $\sim 0.3$ ), then it follows that the pull-off force should be proportional to $F_{\mathrm{N}}^{1 / 3}$. We now present adhesion experiments to test these hypotheses (see also Ref. 46).

Figure 8(a) shows the interaction force as a function of time when a glass ball (diameter $2 R=5 \mathrm{~cm}$ ) approaches and retracts (speed $v_{z}= \pm 25 \mu \mathrm{m} / \mathrm{s}$ ) from the bromobutyl rubber sheet. We show results for three different loading regimes, where the maximum loading forces are $F_{\mathrm{m}}=0.126$ (the red curve), 0.407 (the green curve), and $0.747 \mathrm{~N}$ (the blue curve). Assuming that the rubber elastic modulus $E_{\text {eff }}=2.5 \mathrm{MPa}$ gives the Hertz maximum contact pressures $p_{0}=0.072,0.106$, and $0.130 \mathrm{MPa}$. These are all in the region where, in the absence of adhesion, we expect the contact area to depend linearly
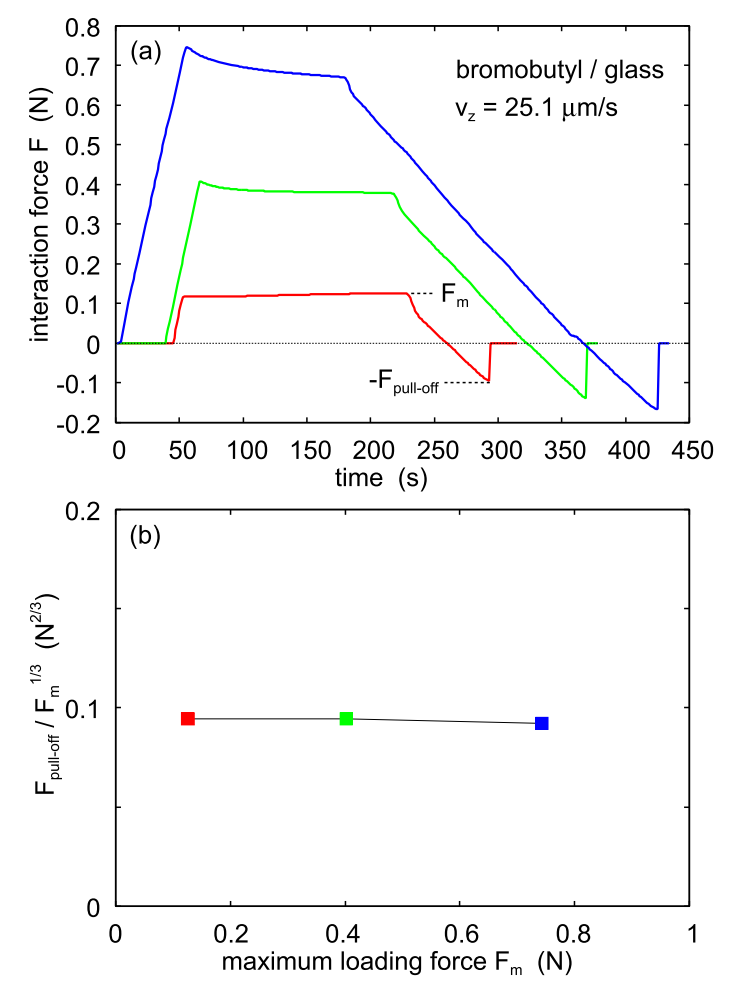

FIG. 8. (a) The interaction force for three different loading regimes as a function of time. The glass ball approach and retraction velocity $v_{z}= \pm 25 \mu \mathrm{m} / \mathrm{s}$.

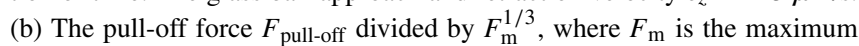
loading force, as a function of $F_{\mathrm{m}}$. For a glass ball with diameter $5 \mathrm{~cm}$ loaded against the bromobutyl rubber.

on the contact pressure (see Fig. 3). Thus, since the Hertz contact pressure scales as $F_{\mathrm{m}}^{1 / 3}$, we expect the pull-off force to be proportional to $F_{\mathrm{m}}^{1 / 3}$. Figure 8 (b) shows that this is indeed the case. Note that for smooth surfaces, where the contact is complete, we do not expect any dependency of the pull-off force on the maximum loading force. This was indeed shown to be the case in Ref. 46 for polydimethylsiloxane (PDMS) rubber with a very smooth surface.

We have performed optical studies of the contact between the bromobutyl rubber and a smooth glass surface, which illustrates the discussion above. Figure 9 shows optical pictures of the contact when the glass plate was first loaded against a rectangular rubber block $(9.0 \mathrm{~mm} \times 6.6 \mathrm{~mm}$ with thickness 2.0 $\mathrm{mm}$ ) with a large force (of order $\sim 50 \mathrm{~N}$ ) for $\sim 5 \mathrm{~s}$, and then removed. The two bright (white) circular area arises from two of the four light diodes used to illuminate the contact.

Due to the strong adhesion hysteresis, after the load was removed the rubber continued to adhere to the glass surface and the contact area was unchanged. Figure 9(a) shows the contact after $\sim 200 \mathrm{~s}$, prior to which we removed (by peeling) the contact between the rubber and the glass surface over the upper half of the nominal contact region. Note the boundary line (opening crack tip) between the non-contact area (the upper half of the picture) and the lower half where (partial) contact occurs.

Figure 9(b) shows the contact after waiting $48 \mathrm{~h}$. Note that the contact in (b) is virtually identical to that obtained immediately after the load was removed [see Fig. 9(a)]. This is a consequence of the huge contact hysteresis prevailing in the present case, where the elastic deformation energy stored 


\section{(a) time $=200 \mathrm{~s}$}

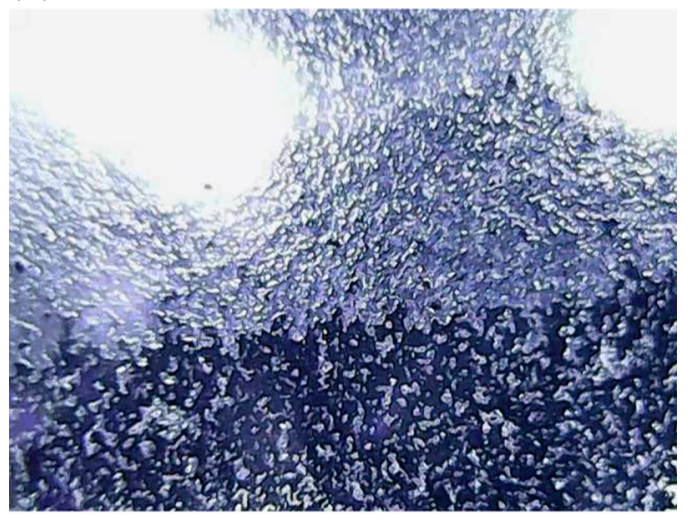

(b) time $=172800 \mathrm{~s}$ (48 hours)

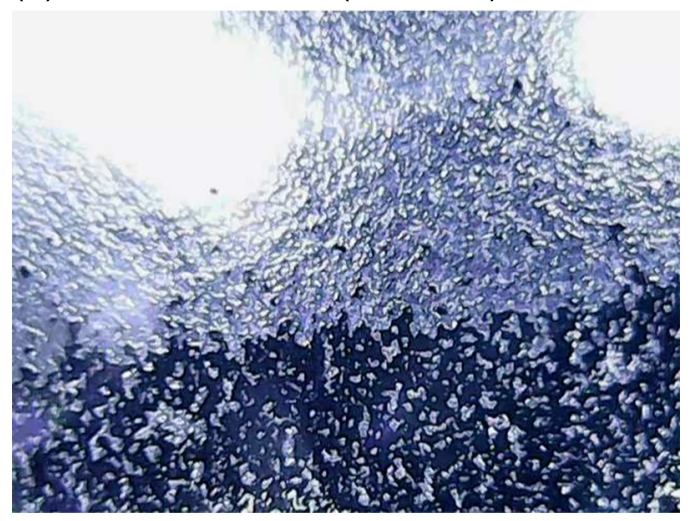

FIG. 9. Optical picture of the contact between the bromobutyl rubber and a flat glass surface. The glass plate was first loaded against the rubber surface with a large force (of order $\sim 50 \mathrm{~N}$ ) for $\sim 5 \mathrm{~s}$ and after the load was removed. We observed that the rubber continues to adhere to the glass surface and the contact area is unchanged. (a) The contact after $\sim 200 \mathrm{~s}$ contact time, prior to which we removed (by peeling) the contact between the rubber and the glass surface over the upper half of the nominal contact region. Note the boundary line (opening crack tip) between the non-contact area and the (partial) contact. (b) After waiting $48 \mathrm{~h}$, the contact is virtually the same as after $\sim 200 \mathrm{~s}$ contact time. This is the result of the huge contact hysteresis: the elastic deformation energy stored in the compressed asperity contact regions is not large enough to propagate opening cracks around the contact spots, resulting in a time-independent contact area.

in the compressed asperity contact regions is not large enough to propagate opening cracks around the contact spots, resulting in a nearly time-independent contact area.

Figure 10 shows the optical picture of the contact between the rubber and the glass surface when the loading force $F_{\mathrm{N}}$ $=8.39 \mathrm{~N}$. The nominal contact pressure $p=F_{\mathrm{N}} / A_{0} \approx 0.1 \mathrm{MPa}$. (a) shows the contact after $200 \mathrm{~s}$ and (b) after $64800 \mathrm{~s} \mathrm{(18} \mathrm{h).}$ The dark regions are contact area. Note that the contact area increases with increasing contact time.

From the optical pictures in Fig. 10, it is possible to estimate the relative contact area, but the result depends on the black/white intensity threshold used: threshold $=0.15$ gives $A / A_{0}=0.14$ after $200 \mathrm{~s}$ and 0.28 after $64800 \mathrm{~s}$. If we instead use threshold $=0.2$, we get 0.18 and 0.35 , respectively, and with the threshold $=0.1$, we get 0.085 and 0.20 , respectively. In all cases, the contact area increases with a factor of $\approx 2$.

There are two effects which result in an increase in the contact area with increasing time. Assume first that adhesion

\section{nominal pressure $0.097 \mathrm{MPa}$}

(a) time $=200 \mathrm{~s}$

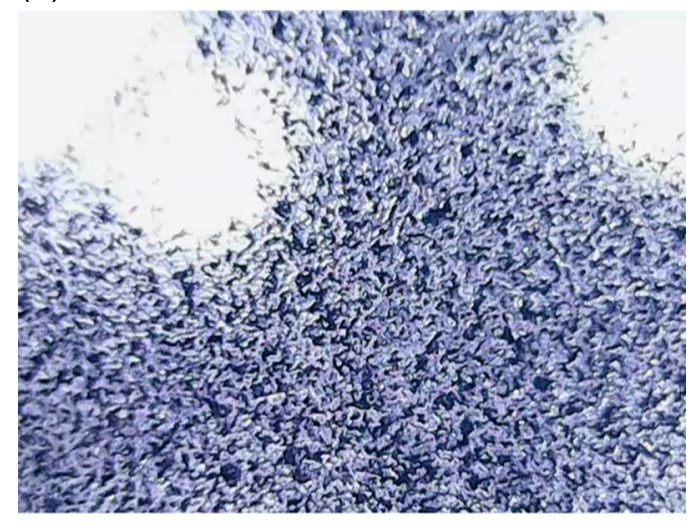

(b) time $=64800 \mathrm{~s}$ (18 hours)

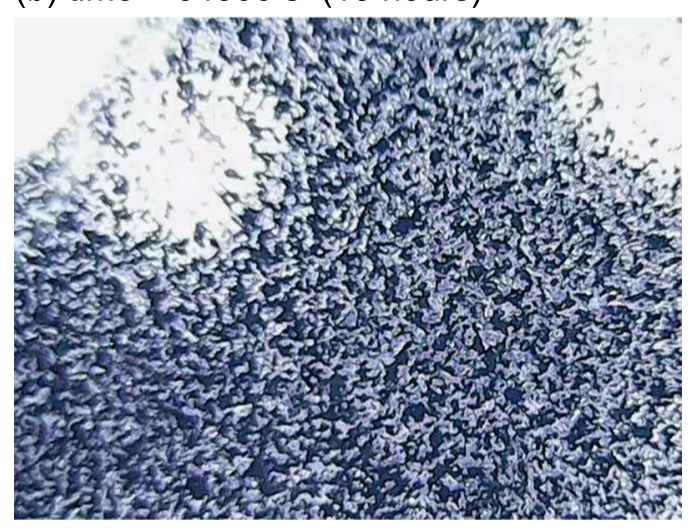

FIG. 10. Optical picture of the contact between the bromobutyl rubber and a flat glass surface loaded against the rubber with the normal force $F_{\mathrm{N}}=8.39$ $\mathrm{N}$. The rubber sample is rectangular with lateral surface area $A_{0}=9.00 \times 9.64$ $\mathrm{mm}^{2}$ and thickness $2 \mathrm{~mm}$. The width of the shown contact region is about $\approx 3$ $\mathrm{mm}$. The nominal contact pressure $p=F_{\mathrm{N}} / A_{0}=0.097 \mathrm{MPa}$. (a) The contact after $200 \mathrm{~s}$. (b) The contact after $64800 \mathrm{~s}$ (18 h). The dark regions are contact area. The two bright (white) circular areas arise from two of the four light diodes used to illuminate the contact. From the optical pictures, it is possible to estimate the relative contact area, but the result depends on the black/white intensity threshold used: threshold $=0.2$ gives $A / A_{0}=0.18$ after $200 \mathrm{~s}$ and 0.35 after $64800 \mathrm{~s}$. If we instead use threshold $=0.15$, we get 0.14 and 0.28 , respectively, and with the threshold $=0.1$ we get 0.085 and 0.20 , respectively. In all cases, the contact area increases with a factor of $\approx 2$.

can be neglected. In this case, the contact area will increase only due to the bulk viscoelasticity. Assuming that the contact area is inversely proportional to the relaxation modulus ${ }^{47,48}$ $E(t)$ (as expected when $A / A_{0} \ll 1$ and without adhesion), from Fig. 1 we get an increase in the contact area with a factor of about 1.3 which is smaller than we observe in the optical experiments.

To understand the role of adhesion, we note first that the asperity contact regions in Fig. 10(a) have diameters of order $30 \mu \mathrm{m}$ which on the average increases by a factor of $\sim 1.4$ when going to Fig. 10(b). Thus the closing crack around each asperity contact region must move with the average speed $v \approx$ $d / t$, where $d \approx 10 \mu \mathrm{m}$ and $t \approx 64800 \mathrm{~s}$, i.e., $v \approx 2 \times 10^{-10} \mathrm{~m} / \mathrm{s}$. For this velocity, from Fig. 11, we obtain the viscoelastic crack reduction factor $1 /[1+f(v, T)] \approx 0.16$. Thus the effective work of adhesion $\gamma_{\text {close }} \approx 0.16 \Delta \gamma \approx 0.045 \mathrm{~J} / \mathrm{m}^{2}$. Using this value from Fig. 3 , we obtain $A / A_{0} \approx 0.35$. This estimation is very rough since the opening crack tip does not move with a constant 


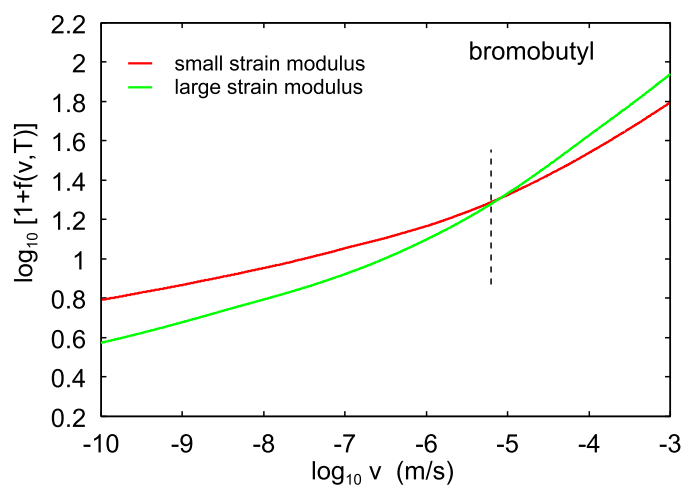

FIG. 11. The viscoelastic crack propagation factor $[1+f(v, T)]$ as a function of the logarithm of the crack tip velocity $v$ for the filled bromobutyl rubber compound at $T=20{ }^{\circ} \mathrm{C}$. The red line uses the low-strain (0.04\% strain) modulus and the green line uses the large-strain $(\approx 15 \%$ strain) modulus. The vertical dashed line indicates a typical crack tip velocity in the experiments reported on in Sec. VI. In the calculations we have used $a_{0}=1 \mathrm{~nm}$ (see Appendix D) and the viscoelastic modulus shown in Figs. 27 and 29.

velocity as assumed above but with a decreasing velocity. Still the estimation shows that the relative contact area estimated by the theory is similar to what is deduced from the optical picture (see Fig. 10).

To estimate more accurately how the contact area depends on the contact time we approximate $w_{\text {open }} \approx w_{0}\left(1+\left(v / v_{0}\right)^{\alpha}\right)$. From Fig. 11 in the relevant (low-velocity) region $\alpha \approx 10$. Thus we get $w_{\text {close }} \approx w_{0} /\left(1+\left(v / v_{0}\right)^{\alpha}\right) \approx w_{0}\left(v / v_{0}\right)^{-\alpha}$. Assuming that each contact region can be treated as a small JKR region, one can show that for long times (or for all times when the nominal contact pressure vanishes and $w_{\text {close }} \sim v^{-\alpha}$ ) the contact area scales with time as $A \sim t^{2 / 3 \alpha}$. When $t$ increases from $t=200 \mathrm{~s}$ to $64800 \mathrm{~s}$, this gives an increase in the contact area with a factor of $(64800 / 200)^{2 / 3 \alpha} \approx 1.5$. Taking that into account as well as the contribution from the viscoelastic relaxation described above, that leads to the total increase by a factor of $1.5 \times 1.3 \approx 2$ which is in good agreement with the observations (see Fig. 10). Note also that $t^{2 / 3 \alpha}$ $=\exp ((2 / 3 \alpha) \log t) \approx 1+(2 / 3 \alpha) \log t$ as long as $(2 / 3 \alpha) \log t$ $\ll 1$ so in some intermediate time interval the relation $A / A_{0}$ $\sim t^{2 / 3 \alpha}$ is similar to a logarithmic time-dependency.

We have shown above that when strong contact hysteresis occurs the work of adhesion and hence the JKR pull-off force are proportional to the normalized area of real contact,

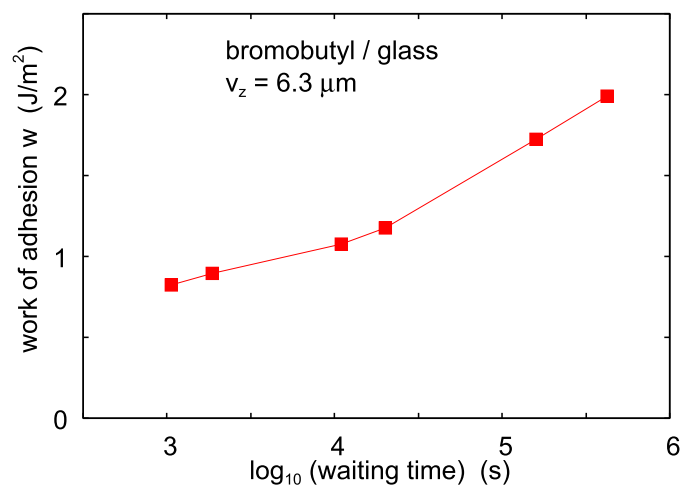

FIG. 12. The experimentally measured work of adhesion as a function of the logarithm of the waiting contact time. For a glass ball (diameter $2 R=5 \mathrm{~cm}$ ) against the bromobutyl rubber. The maximal normal load $F_{\mathrm{m}} \approx 0.2 \mathrm{~N}$.
$A / A_{0}$. The contact area increases with the time of stationary contact due to viscoelastic relaxation (see Fig. 1), and (more importantly) due to strengthening of the adhesive interaction with increasing contact time.

The effect of the contact time on the pull-off force is illustrated in Fig. 12 where we show the measured work of adhesion (red squares) as a function of the logarithm of the waiting contact time (see Sec. V for the experimental details). The pull-off force increases by a factor of $\sim 2.2$ as the contact time increases from $10^{3} \mathrm{~s}$ to $3 \times 10^{5} \mathrm{~s}$. This is in good agreement with the timedependency of the contact area observed above (see Fig. 10) and, as explained above, is probably mainly due to the velocity dependency of the work of adhesion for the closing crack tip.

\section{ADHESION: EXPERIMENTS AND ANALYSIS}

In this section, we describe the set-up used for our adhesion studies and present results for the contact between smooth glass and the bromobutyl rubber in dry condition, and when immersed in water and in silicone oil.

\section{A. Experimental}

We study the adhesion interaction between spherical silica glass balls [diameter $2 R=2.5 \mathrm{~cm}$ (in fluids), and $4 \mathrm{~cm}$ or $5 \mathrm{~cm}$ (dry state)] and rubber in dry and lubricated conditions. We bring the ball into contact with the substrate using a drive which can be represented by a spring (see Fig. 33(b) and Appendix H). The contact region is not observed directly but only the time dependency of the interaction force $F(t)$ and the displacement $s(t)$ of the upper part of the driving spring are measured. The experimental adhesion data are analyzed using the JKR theory (see Appendix E).

The rubber substrate is positioned on a very accurate balance (analytical balance produced by Mettler Toledo, model MS104TS/00) which has a reproducibility of $0.1 \mathrm{mg}$ ( or $\approx 1$ $\mu \mathrm{N}$ ) (see Fig. 13). After zeroing the scale of the instrument, we can measure the force $F(t)$ on the substrate as a function of time, which is directly transferred to a computer at a rate of 1 or 10 measurement points per second.

To move the glass ball up and down, we use an electric motor coiling up a nylon cord, which is attached to the glass ball. The drive velocity as a function of time can be specified on a computer. In the experiments reported on below, the glass ball is repeatedly moved up and down, sometimes for more than 10 contact cycles, involving a measurement time

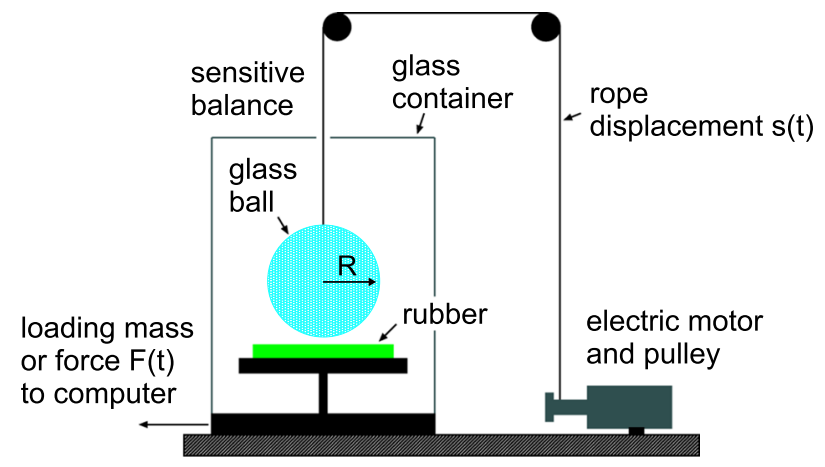

FIG. 13. The experimental set-up for measuring adhesion. 
of sometimes more than $24 \mathrm{~h}$. The maximum loading force is typically $\sim 0.1 \mathrm{~N}$ corresponding to the loading mass $\sim 10 \mathrm{~g}$.

We consider the adhesion between borosilica glass balls and the (carbon black filled) bromobutyl rubber. Here we note that the rubber contains low-molecular weight components, which can migrate (diffuse) from the bulk to the surface. In the experiments, we have used rectangular or circular rubber sheets with a diameter of order a few $\mathrm{cm}$, and the thickness $\approx 2 \mathrm{~mm}$. For measurement of adhesion in the dry state, the rubber sheets were attached to a flat surface using double sided adhesive tape. For measurements in liquids, the rubber sheets were confined at the bottom of a cylindrical PMMA tube using an elastic spring.

We study first the dry rubber-glass contact, where the rubber surface has been cleaned using different procedures. Next we study the adhesion when the system is immersed in water or in silicone oil.

\section{B. Adhesion for dry condition}

The adhesion experiments consist of several loadingunloading cycles. In the loading phase, we lower the glass ball toward the rubber sample with a constant speed $v_{z}$. After some fixed displacement, which results in the contact between the glass ball and the rubber (contact force $\sim 0.1 \mathrm{~N}$ ), we reverse the velocity and pull-off the glass ball with the same velocity as during approach. As an example, in Fig. 14 we show the interaction force between the glass ball and the rubber compound as a function of time for 4 contact cycles with the drive speed $v_{z}= \pm 0.9 \mu \mathrm{m} / \mathrm{s}$. The green line is for bromobutyl cleaned by brushing the surface with soft toothbrush in boiling hot water for about $1 \mathrm{~min}$. The blue curve is the same procedure followed by lapping the rubber surface for a few second with soft tissue wet by acetone.

Note that the detachments in Fig. 14 occur rather abruptly (in about $\sim 1 \mathrm{~s}$ ). In fact, for a purely elastic solid and neglecting inertia the detachment time would be zero, but in the present case the detachment time is determined by the velocity dependency of the crack propagation energy.

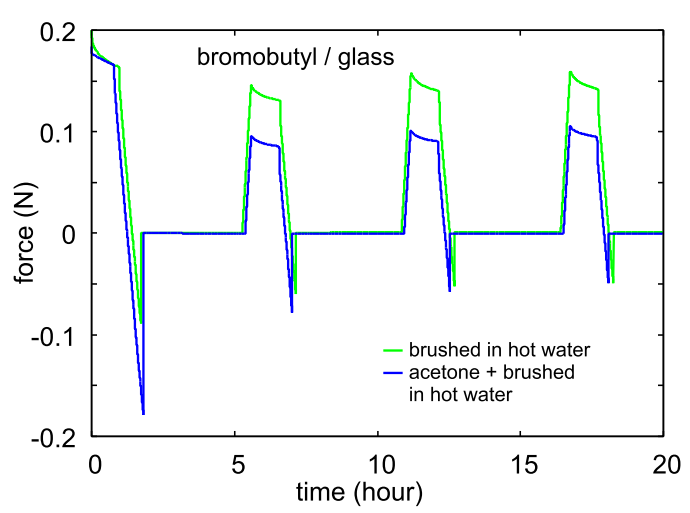

FIG. 14. The interaction force between the glass ball and the bromobutyl rubber as a function of time for 4 contact cycles with the drive speed $v_{z}=0.9$ $\mu \mathrm{m} / \mathrm{s}$. The green line is for the rubber cleaned by brushing the surface with soft toothbrush in boiling hot water for about $1 \mathrm{~min}$. The blue curve is the same procedure, but first the rubber surface was cleaned by lapping it for a few second with soft tissue wet by acetone.

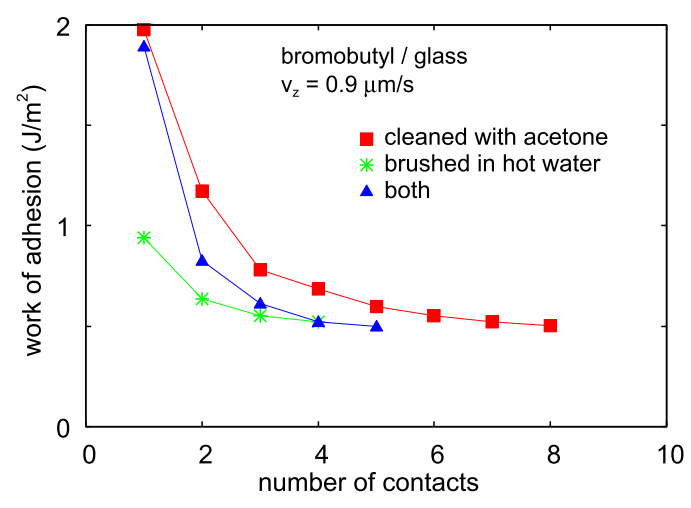

FIG. 15. The work of adhesion during retraction (separation) as a function of the number of contacts between bromobutyl rubber with surfaces. The glass ball was originally cleaned with acetone, and the rubber surfaces were cleaned with acetone (squares), brushed in hot distilled water (star), or first cleaned in hot water and then with acetone (triangles). Note that the adhesion decreases with the number of contacts due to transfer of molecules from the rubber to the glass surface.

Figure 15 shows the work of adhesion during retraction (separation) as a function of the number of contacts. The glass ball was originally cleaned with acetone and the rubber surfaces with acetone (square symbols) or brushed in hot distilled water (stars), or first cleaned in hot water and then with acetone (triangles). Note that in all cases, due to transfer of mobile molecules (e.g., stearic acid) from the rubber surface to the glass surface, the adhesion decreases with the number of contacts.

A decrease in the work of adhesion with increasing number of contacts has also been observed in Refs. 49-52 for sapphire and glass balls in contact with silicone rubber. In these cases, the reduction in adhesion was attributed to transfer of oligomers from the rubber to the balls.

Figure 16 shows the result of a second adhesion study performed $\approx 1 / 2$ year after the experiments reported on in Fig. 15, and for a higher the pull-off speed $(5 \mu \mathrm{m} / \mathrm{s}$, as compared to $0.9 \mu \mathrm{m} / \mathrm{s}$ in Fig. 15). In this case, the rubber surfaces were cleaned by lapping it for a few second with soft tissue wet by isopropanol. Isopropanol is a "softer" cleaning fluid than acetone, but the results are very similar.

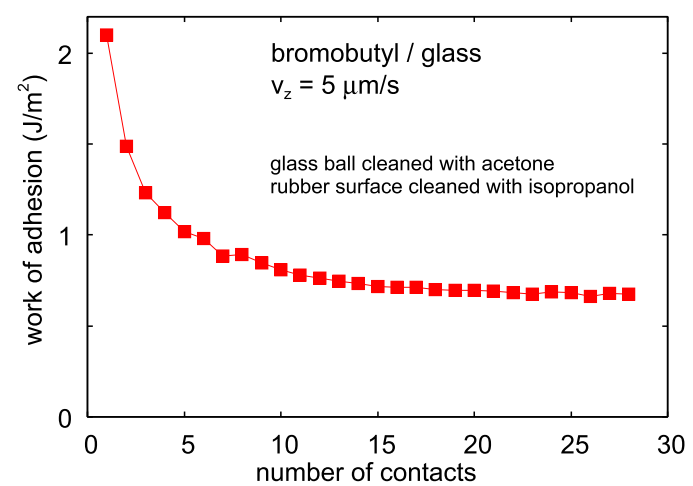

FIG. 16. The work of adhesion during retraction (separation) as a function of the number of contacts between bromobutyl rubber with surfaces. The glass ball was originally cleaned with acetone, and the rubber surfaces were cleaned with isopropanol. The experiment was performed $\approx 1 / 2$ year after the experiments reported on in Fig. 15. Note that the adhesion decreases with the number of contacts due to transfer of molecules from the rubber to the glass surface. 
Figure 11 shows that for the crack tip velocity $v_{r}=5 \mu \mathrm{m} / \mathrm{s}$, the viscoelastic enhancement factor for the work of adhesion is $(1+f) \approx 20$. If we assume a clean glass surface and that only dispersion forces act between the rubber and the glass surface, the adiabatic work of adhesion was estimated in Sec. II to be $\Delta \gamma \approx 0.1 \mathrm{~J} / \mathrm{m}^{2}$, and if we use the contact area $A_{1} / A_{0}$ $\approx 0.3$ (see Figs. 3 and 11 ) we get the work of adhesion $w \approx \Delta \gamma\left(A / A_{0}\right)(1+f) \approx 0.6 \mathrm{~J} / \mathrm{m}^{2}$. This is a factor of $\sim 3$ smaller than what is observed in Figs. 15 and 16 for the first contact with the glass ball. Hence for the clean glass ball the work of adhesion $\Delta \gamma \approx 0.3 \mathrm{~J} / \mathrm{m}^{2}$. This is larger than expected in the adiabatic limit if only dispersion forces act between the rubber and the glass surface (see Sec. II) and shows that some other types of stronger bonds form between the clean glass and rubber surfaces. However, after the glass surface is contaminated the work of adhesion drops to $\approx 0.6 \mathrm{~J} / \mathrm{m}^{2}$ and at this point the dispersion forces may give the dominant contribution to the work of adhesion.

\section{Adhesion in water}

We have performed adhesion experiments with the glass ball and rubber immersed in water and in silicone oil. Figure 17 shows the experimental set-up. The fluid is located in a PMMA container (inner diameter $4 \mathrm{~cm}$ ) with a PMMA cover to avoid evaporation of the fluid. The top cover has a small hole (diameter $1 \mathrm{~mm}$ ) through which the nylon rope (diameter 0.3 $\mathrm{mm}$ ), used for moving the glass ball, passes. The rubber sheet is located at the bottom of the container. The glass ball (diameter $2 R=2.5 \mathrm{~cm}$, with a flattened top part) is fully immersed in the fluid during the contact cycling. The container is located on a sensitive laboratory balance used for measuring the pull-off force.

Figure 18 shows the work of adhesion during retraction (separation) as a function of the number of contacts between

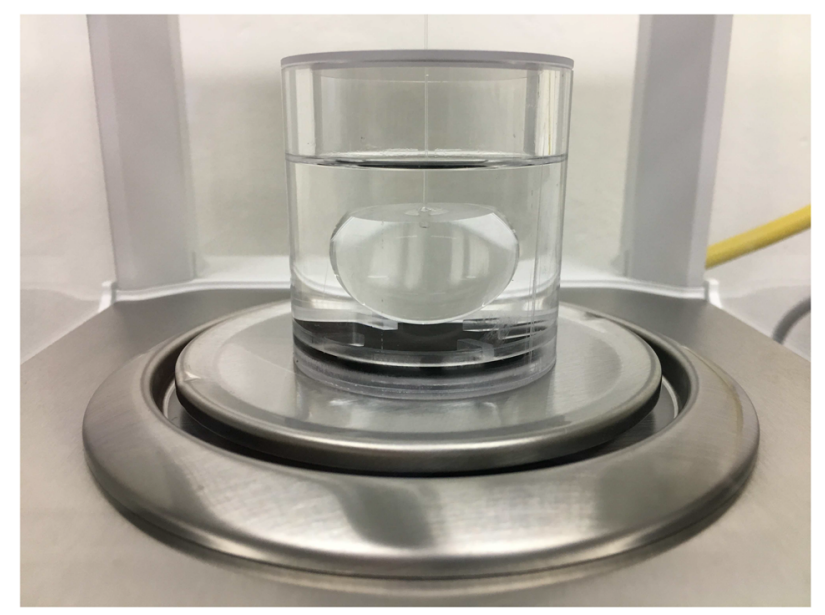

FIG. 17. Experimental set-up for adhesion studies in fluids (in the case of silicone oil). The fluid is located in a PMMA container (inner diameter $4 \mathrm{~cm}$ ) with a PMMA's cover to avoid evaporation of the fluid. The top cover has a small hole (diameter $1 \mathrm{~mm}$ ) through which the nylon rope (diameter $0.3 \mathrm{~mm}$ ), used for moving the glass ball, passes. The rubber sheet (in this case a carbon filled bromobutyl rubber sheet) is located at the bottom of the container. The glass ball (diameter $2.5 \mathrm{~cm}$, with a flattened top part) is fully immersed in the fluid during the contact cycling. The container is located on a sensitive laboratory balance used for measuring the pull-off force.

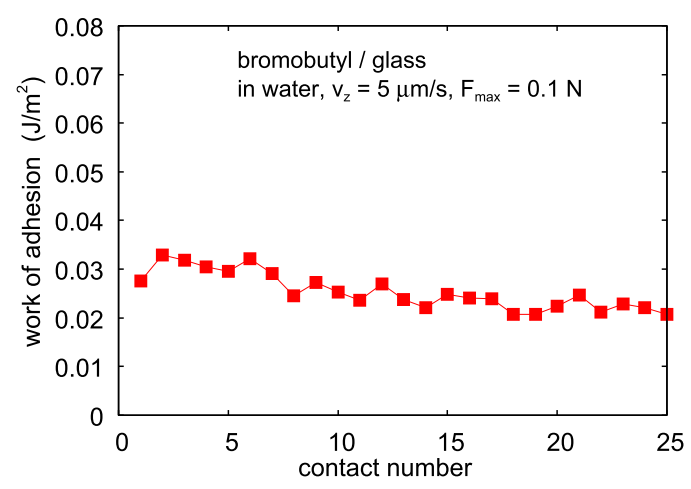

FIG. 18. The work of adhesion during retraction (separation) as a function of the number of contacts between the glass ball (diameter $2 R=2.5$ $\mathrm{cm}$ ) and the bromobutyl rubber in water. The glass ball was originally cleaned with acetone and isopropanol. The rubber surface was cleaned with isopropanol.

the glass ball and the rubber surface. The glass ball was originally cleaned with acetone and isopropanol. The rubber surface was cleaned with isopropanol. The red squares are for the case the glass ball made contact with the rubber in water. Note the drop in the work of adhesion with increasing number of contacts, which is much smaller than for the dry contact (Figs. 15 and 16). Thus there appears to be a strong reduction in the transfer of molecules from the rubber to the glass surface when the contact is in water as compared to the dry contact.

Figure 19 shows the work of adhesion during retraction (separation) as a function of the contact time between the glass ball and the rubber surface. Note the huge increase in the work of adhesion with increasing contact time. This increase is much stronger than observed for dry surfaces, where the work of adhesion increased with a factor of $\sim 1.5$ as time increases from $10^{3} \mathrm{~s}$ to $10^{5} \mathrm{~s}$ (see Fig. 12). In the present case, the increase in the same time interval is a factor of $\sim 5$. We interpret this as resulting from (slow) thermally activated dewetting transitions in the asperity contact regions. We have seen in Sec. III that continuum mechanics calculations for the contact between the glass ball and the rubber in water predict that already after $\sim 1 \mathrm{~s}$ the water is squeezed out from the asperity contact regions, and the contact area and the interfacial

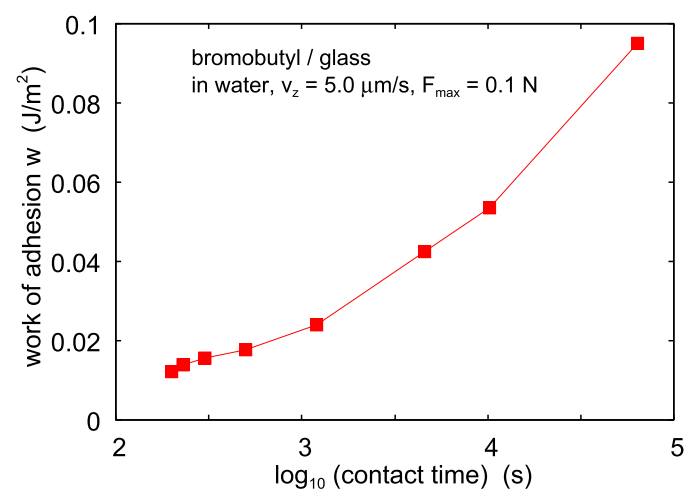

FIG. 19. The work of adhesion during retraction (separation) as a function of the contact time between the glass ball and the bromobutyl surface in water. The glass ball was originally cleaned with acetone and isopropanol. The rubber surface was cleaned with isopropanol. 
separation become time independent. This is also consistent with optical pictures of the contact in water where the contact looks the same after $\sim 10 \mathrm{~h}$ as compared to $\sim 100 \mathrm{~s}$ contact time. However, we believe that there is a very thin (of order nanometer) water film between the rubber asperities and the glass surface, which only very slowly is removed by the nucleation of dry rubber-glass surface areas (dewetting transitions). Only by assuming this is it possible to explain the observed strong increase in the work of adhesion with increasing contact time (see Fig. 19).

We have argued in Sec. II that the interaction potential between rubber and the glass surface in water has the form shown in Fig. 2. At short separation, the interaction potential has a local minimum corresponding to direct contact between the rubber and the glass surface. In this case, bonding forces (of unknown nature), stronger than the dispersion forces, occur between the surfaces.

When a bromobutyl rubber is squeezed in contact with the glass surface in water we expect first a rapid squeeze-out (see Sec. III) until contact occurs between the rubber asperities and the glass surface. However, at short contact time we do not expect any true atomic contact between the rubber and the glass surface, but a water film of nanometer thickness separates the surfaces in the asperity contact regions. However, this state is metastable and after a long enough time we expect true atomic contact to form between the rubber and the glass surface in the asperity contact regions. This dewetting process involves the nucleation (by thermal fluctuations) of nanometer sized contact regions followed by the removal of the nanometer water film by a (mainly) surface-energy driven squeeze-out process. Thus we expect the contact area between the rubber and the glass surface to increase continuously with increasing contact time, which is consistent with the observed increase in the work of adhesion with increasing contact time (see Fig. 19). We note, however, that optical pictures of the glass-rubber contact in water do not show any time-dependent changes. This is due to the fast fluid squeeze-out and to the limitation of our optical microscope. Thus, with our optical set-up, if the rubber-glass surfaces are separated by thin fluid film, say, $1 \mathrm{~nm}$ of water, it may appear the same as for direct rubber-glass contact.

Figure 20 shows an optical picture of the contact between the glass and rubber surface squeezed together in water. Note the granular structure which must be caused by the rubber surface roughness. Similar pictures obtained after different contact times (not shown) look the same.

Figure 21 shows an optical picture of a small water droplet squeezed between the glass and rubber surface. Both dry and wet regions can be observed. When the normal load is slowly increased, the fluid covered region increases in size via small local (rapid) expansions of the wet area, followed by time periods where no change occurs in the line boundary separating the water covered and the dry surface area. We interpret this as a Laplace-pressure effects, where the water pressure locally must become high enough to overcome surface-energy derived energetic barriers associated with narrow constrictions in the non-contact fluid flow channels. These energy barriers could be important for the fluid flow at the interface in leakage experiments when a small applied fluid pressure

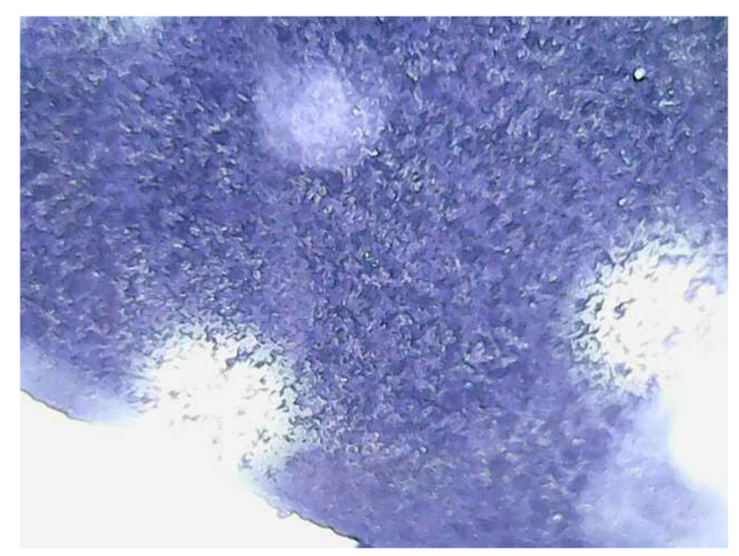

FIG. 20. Optical picture of the contact between a flat glass plate and a rectangular rubber block squeezed together in water. Note the granular structure which results from the rubber surface roughness. For the glass-rubber contact fully immersed in water, we cannot detect any time-dependent changes in the optical pictures. This is due to the rapid fluid squeeze-out and the fact that in the optical pictures the rubber-glass contact regions where the surfaces are separated by, say, $1 \mathrm{~nm}$ of water, look the same as when the rubber is in direct contact with the glass.

difference occurs between the two sides in a seal (see Refs. 12 and 53).

Note that if the water is completely removed from some rubber-glass contact regions during the loading phase, during pull-off, because of the strong adhesion hysteresis, the effective work of adhesion may be large. When strong contact hysteresis occurs we have shown in Sec. V that $w$ $=\gamma_{\text {open }}(v) A / A_{0}$. The large magnitude of $\gamma_{\text {open }}(v)=\Delta \gamma(1+f)$ implies that the relative contact area $A_{1} / A_{0}$ must be very small for the contact in water for short contact times. Thus using $\gamma_{\text {open }}(v) \approx 1 \mathrm{~J} / \mathrm{m}^{2}$ and $w=0.01 \mathrm{~J} / \mathrm{m}^{2}$ as observed for very short contact time (about $200 \mathrm{~s}$ ), we get $A / A_{0} \approx 0.01$ as compared to $A / A_{0} \approx 0.3$ for the dry contact case. However, the relative contact area increases monotonically due to dewetting in the asperity contact regions and for $t \approx 10^{5} \mathrm{~s}$ from Fig. 19 we have $w \approx 0.1 \mathrm{~J} / \mathrm{m}^{2}$ and hence $A / A_{0} \approx 0.1$.

On contact formation (approach) in water (and in silicon oil), no adhesion can be detected, while during pull-off we always observe adhesion.

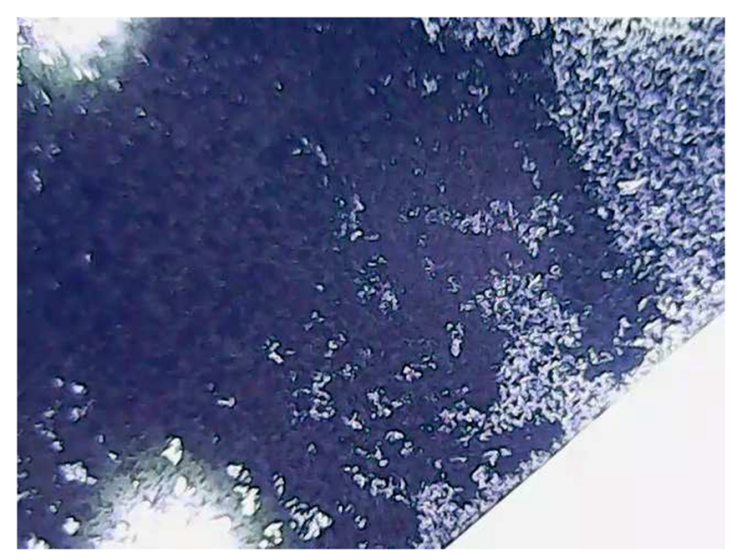

FIG. 21. An optical picture of a small water droplet squeezed between a glass plate and a rectangular rubber block. Both dry and wet regions can be observed but pictures obtained at different times look the same in the wet region. 


\section{Adhesion in silicone oil}

We have performed two sets of experiments involving silicone oil. In one experiment, the rubber and the glass ball was covered with thin films (thickness $d$ ) of silicone oil. In this case, we observed adhesion due to the formation of a capillary bridge (see Appendix G). In the second set of experiments, which we report on here, the glass ball and the rubber sample is fully immersed in the silicone oil.

Figure 22 shows the work of adhesion as a function of the contact number between the glass ball and the bromobutyl rubber immersed in silicone oil (viscosity $0.35 \mathrm{~Pa} \mathrm{~s}$ ). The results are shown for the pull-off speeds $0.9,1.8,6.3$, and $35 \mu \mathrm{m} / \mathrm{s}$. Note the strong dependency of the work of adhesion on the pull-off speed in contrast to the dry case where the change in the work of adhesion with the pull-off speed is very weak. In all cases, the "work of adhesion" is very small, and, in Appendixes H-J, we show that it is strongly influenced by the viscosity of the oil. Thus, at least for the two highest pull-off speeds, the JKR theory is probably not valid, and, in these cases, the work of adhesion must be interpreted as just the pull-off force $F_{\mathrm{c}}$ divided by $(3 \pi / 2) R$, i.e., it cannot be considered to be an effective binding energy per unit surface area. This fluid-viscosity effect is negligible in water because water has 350 times smaller viscosity than the silicone oil (see Appendix J).

For pull-off in the dry state and in water, we observed very rapid detachment transition involving a transition time of order $\sim 1 \mathrm{~s}$. For the system immersed in silicone oil, the detachment transition occurs over much longer time period. This is illustrated in Fig. 23, which shows the interaction force as a function of time close to a detachment for (a) dry surfaces, (b) in water, and (c) in silicone oil. The drive speed is $v_{z}=0.9 \mu \mathrm{m} / \mathrm{s}$ in (a) and (b) and $1.8 \mu \mathrm{m} / \mathrm{s}$ in (c). In (c), we have superimposed (by shifting along the time axis) 4 different pull-off events indicated by the different symbols. The data presented in Fig. 23 were collected at a rate of one data point per second, and the detachment transitions in (a) and (b) clearly occur within $1 \mathrm{~s}$, while in case (c) it extends over more than $10 \mathrm{~s}$.

The green line in (c) is the theory prediction for the interaction force assuming only a viscous drag force (see Appendix I),

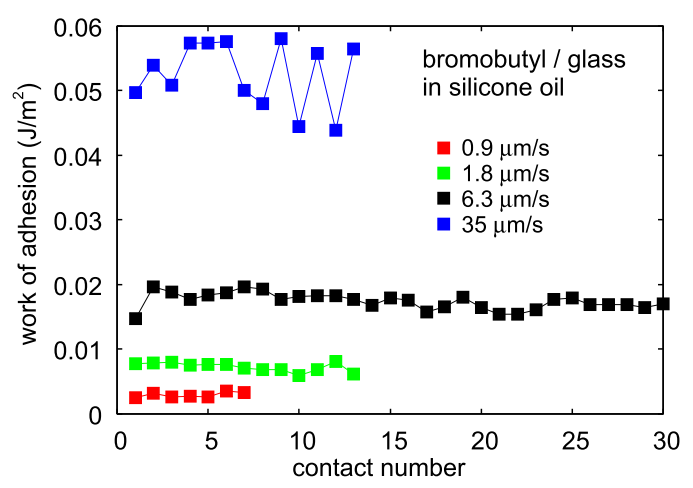

FIG. 22. The work of adhesion as a function of the contact number for the glass ball and the bromobutyl rubber immersed in silicone oil (viscosity 0.35 Pa s). The results are shown for the pull-off speeds, $0.9 \mu \mathrm{m} / \mathrm{s}$ (red squares), 1.75 $\mu \mathrm{m} / \mathrm{s}$ (green squares), $6.3 \mu \mathrm{m} / \mathrm{s}$ (black squares), and $35 \mu \mathrm{m} / \mathrm{s}$ (blue squares).
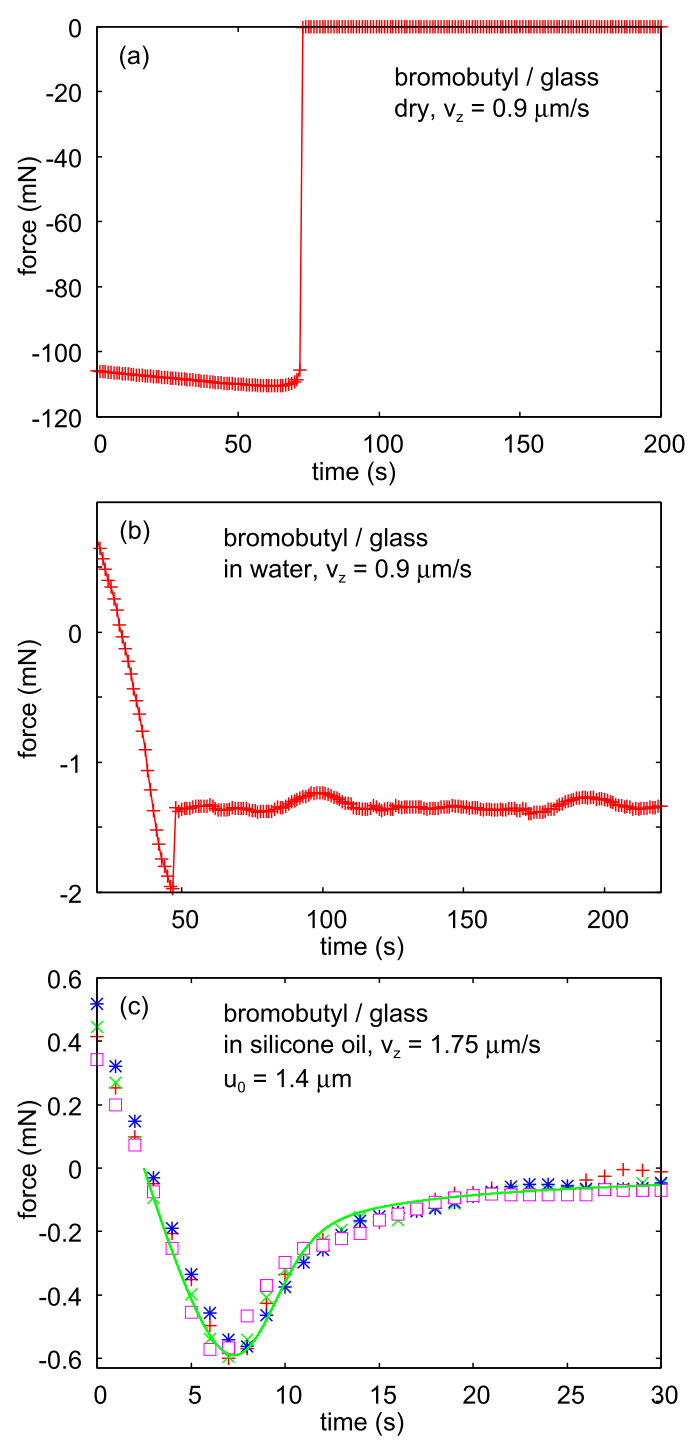

FIG. 23. The interaction force between the glass ball and the rubber as a function of time close to a detachment for (a) dry surfaces, (b) in water, and (c) in silicone oil (viscosity $0.35 \mathrm{~Pa} \mathrm{~s}$ ). The drive speed is $v_{z}=0.9 \mu \mathrm{m} / \mathrm{s}$ in (a) and (b) and $1.8 \mu \mathrm{m} / \mathrm{s}$ in (c). In (c), we have superimposed (by shifting along the time axis) 4 different pull-off events indicated by the different symbols. The green line in (c) is the theory prediction for the interaction force assuming only a viscous drag force (see Appendix I), with the initial (at $F=0$ ) surface separation $u_{0}=1.4 \mu \mathrm{m}$.

with the initial (at $F=0$ ) surface separation $u_{0}=1.4 \mu \mathrm{m}$. Thus viscous effects appear to explain the dependency of the interaction force on time when $F(t)<0$. However, the initial separation (at $F=0$ ) $u_{0}$ is determined by adhesion since without adhesion theory predicts much larger surface separation at $F=0$ (see Appendix F). This is also consistent with the results obtained in Sec. VI E, which shows that the pull-off force depends on the chemical status of the glass surface which would not be the case if only the fluid viscosity would affect the adhesion.

In Appendix J, we show that the average surface separation $u_{0}$ when $F=0$ decreases as the pull-off speed decreases. Thus we find $u_{0}=1.8,1.65$, and $1.4 \mu \mathrm{m}$ for $v_{z}=35,6.3$, and $1.75 \mu \mathrm{m} / \mathrm{s}$, respectively. We interpret this as resulting from the longer glass-rubber contact time at the lower approach and pull-off speed (the contact time is proportional to $1 / v_{z}$ ). 
As a further test, we performed another experiment with the pull-off velocity $6.3 \mu \mathrm{m} / \mathrm{s}$ where we kept the ball in contact with the rubber for $7200 \mathrm{~s}$ before removing the contact. In this case, we found that using $u_{0}=1.3 \mu \mathrm{m}$ gives good agreement with the experiment, see Fig. 41 . This is considerable smaller than $1.65 \mu \mathrm{m}$ found without the waiting time period. This is in accordance with the results obtained in Sec. VI E, where we found that for the clean glass ball the longer contact time results in a stronger adhesive interaction between the glass ball and the rubber, and hence to a smaller $u_{0}$.

Here we also note that for the rubber-glass contact in water, which exhibits a similar work of adhesion as in silicone oil, optical microscope pictures of the rubber-glass contact do not show any change when the loading force changes from its maximum to zero. This again indicates that the energy to propagate the opening crack (at relevant speeds) is so large that the compressed asperities cannot reduce the contact area during unloading.

\section{E. On the dependency of the pull-off force on the contact time in silicone oil}

In Sec. VID, we showed that the pull-off force for the contact of the glass ball and the rubber in water increases strongly with increasing time of contact. We interpreted this as resulting from a slow (thermally activated) dewetting process, where a nanometer water film is removed from the asperity contact regions. Here we study the same for the contact in silicone oil for bare glass and for glass passivated by baked-on silicone oil.

Glass surfaces with baked-on silicone oil exhibit low friction and low adhesion against rubber. In our case, the glass balls were first cleaned by ultrasonic in purified water. Baked-on siliconisation involves the application of silicone oil (usually as an emulsion) on a glass surface, which then is baked on to the glass surface at a specific temperature and for a specific time period. We use an aqueous siliconization emulsion that contain 35\% Dow Corning 360 Medical Fluid, 350 sSt (see Ref. 54). The silicone oil emulsion is sprayed on the glass ball.

In the baked-on process, hydrogen and covalent bonds form between the glass surface and the polydimethylsiloxane chains (see Fig. 24). This results in a very inert and hydrophobic coating, where the bonds are so strong that the attached polydimethylsiloxane chains cannot be removed with solvent.
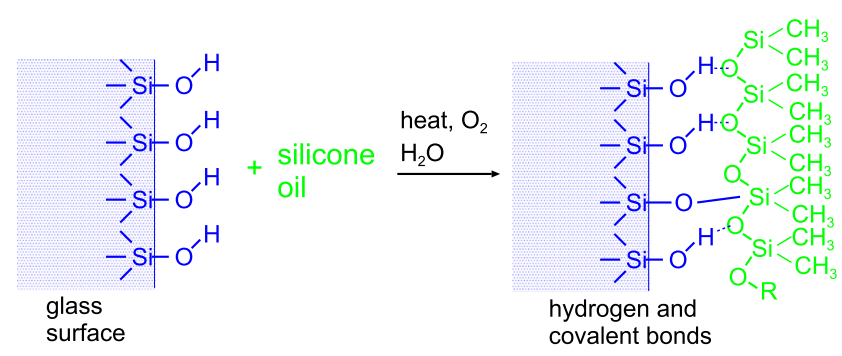

FIG. 24. Baked-on siliconisation involves the application of silicone oil (usually as an emulsion) on a glass surface, which then is baked onto the glass surface at a specific temperature and for a specific time period. In the bakedon process, hydrogen and covalent bonds form between the glass surface and the polydimethylsiloxane chains. This results in a very inert and hydrophobic coating, where the bonds are so strong that the attached polydimethylsiloxane chains cannot be removed with solvent.

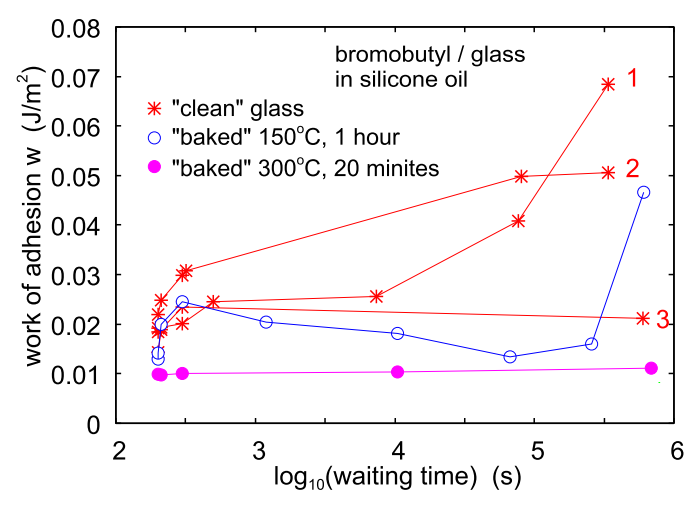

FIG. 25. The work of adhesion as a function of the logarithm of the waiting time for a "clean" glass ball (red stars) and for a glass ball with baked-on silicone oil using procedure 2 (blue open circles) and for glass ball with bakedon silicone oil obtained using procedure 1 (pink filled circles). For contact between glass balls with diameter $2 R=2.5 \mathrm{~cm}$ and the bromobutyl rubber in silicone oil (viscosity $0.35 \mathrm{~Pa} \mathrm{~s}$ ). The pull-off velocity $v_{z}=6.28 \mu \mathrm{m} / \mathrm{s}$. The numbers 1-3 for the "clean" glass balls indicate the time-order of the measurements where increasing number corresponds to later measurement during a total time interval of $\sim 5$ month. During the waiting time, the glass balls were immersed in silicone oil.

One set of balls was baked at $T=300{ }^{\circ} \mathrm{C}$ for $20 \mathrm{~min}$ (procedure 1) according to the standard industrial procedure for siliconisation of glass surfaces. Another set of balls was baked at $T=150{ }^{\circ} \mathrm{C}$ for $1 \mathrm{~h}$ (procedure 2). In this latter case, the balls were cleaned prior to the baking by exposing the glass balls to oxygen plasma.

Figure 25 shows the work of adhesion as a function of the logarithm of the waiting time for a "clean" glass ball (red stars) and for glass balls baked in silicone oil using procedure 1 (filled circles) and procedure 2 (open circles). The glass balls were removed with the speed $v_{z}=6.28 \mu \mathrm{m} / \mathrm{s}$.

For short times, all the glass balls give a similar effective work of adhesion. We interpret this (see also above and Appendix H) as due mainly to the viscous drag which is the same in all cases. Thus, we expect the surfaces in most of the asperity contact regions to be separated by one or several monolayers of silicone oil molecules. We note, however, that the adhesion must be present since otherwise the (average) interfacial separation when the force is reduced to zero, $F=0$, would be so large as to give much smaller viscous pull-off forces than observed (see Appendix F).

In some cases, the work of adhesion for times $>10^{4} \mathrm{~s}$ increases very rapidly. This may involve dewetting transitions where the silicone oil is completely removed from some asperity contact regions. This is most likely a thermally activated process and needs long times to manifest.

The numbers 1-3 for the "clean" glass balls indicate the time-order of the measurements, where increasing number corresponds to the later measurement during a total time interval of $\sim 5$ month. During the waiting time, the glass balls were immersed in silicone oil. Clearly some type of (thermally activated) aging has occurred, which has resulted in a decrease in the adhesive force with increasing time in contact with the oil. We attribute this to an increasing passivation of the glass surface involving processes similar to what is shown in Fig. 24. Experiments performed with new rubber and new silicone oil after long waiting time give a very low work of adhesion 


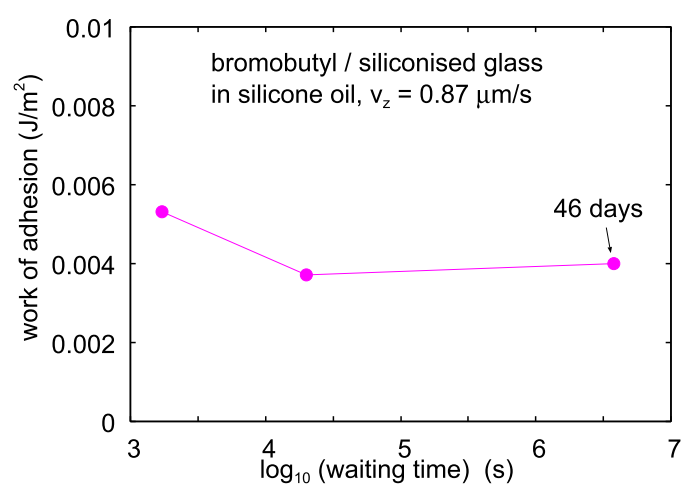

FIG. 26. The work of adhesion as a function of the logarithm of the waiting time for a glass ball with baked silicone oil produced using procedure 1 . The pull-off speed $v_{z}=0.87 \mu \mathrm{m} / \mathrm{s}$.

indicating that the "aging" probably relates to passivation of the glass ball.

Note in Fig. 25 the strong increase in $w$ for waiting times $t>10^{4} \mathrm{~s}$ for the (original) clean glass ball during the first test (denoted as 1). This agrees with the time dependency of the break loose friction force for syringes with the glass barrel, where the contact between the rubber stopper and the glass is lubricated with silicone oil with similar viscosity as used above (see Ref. 13). In Ref. 13, it was suggested that the increase in the break loose friction force is associated with a dewetting transition.

Figure 26 shows the work of adhesion as a function of the logarithm of the waiting time for $v_{z}=0.87 \mu \mathrm{m} / \mathrm{s}$. Again the work of adhesion does not depend on the contact time and is roughly a factor of 2 smaller than for the higher pull-off speed used in Fig. 25. This reduction in the work of adhesion (or rather the pull-off force) is however much smaller than what would be expected if the pull-off force would be determined by the viscosity of the fluid since the velocity is reduced by a factor of $0.87 / 6.28 \approx 0.14$. We attribute this to an adhesioninduced reduction in the average interfacial separation $u_{0}$ (at the time when $F=0$ ) due to the longer contact time at the lower pull-off speed.

\section{SUMMARY AND CONCLUSION}

Adhesive systems of glass in contact with filled bromobutyl rubber in dry conditions, in water, and in silicone oil have been experimentally investigated. The experimental results have been analyzed taking into consideration the interplay between the viscoelastic energy dissipation, at the contact crack tip and surface roughness, and capillary effects and viscous drag in the case of liquid environments. Adhesion hysteresis has been found both in pull-off force tests and supported by optical contact observation, which manifests itself as a strong dependence of the pull-off force (and effective work of adhesion) on the maximum loading force and contact time. For the systems with liquid environments, much weaker adhesion has been observed compared to the dry case. However, the adhesion in liquid is significantly increased with contact time (contact aging), which can be explained by expelling the liquid from the contact region (dewetting transition). Since the latter can be a practically important adverse effect for the silicone-oil lubricated devices, it has been here shown that the glass surface can be protected against the contact aging by the siliconisation process prior to the adhesive contact.

To summarize, the most important results of our study are

(a) For the bromobutyl rubber in contact with a clean glass surface, we observe huge contact hysteresis where the contact area remains unchanged as the external loading force (or squeezing pressure) is removed. We have shown that the contact hysteresis is mainly due to the rubber viscoelasticity, which results in an interfacial crack propagation energy which is much larger for an opening crack than for a closing crack.

(b) For dry rubber-glass contact, we have shown that the contact area increases slowly with time due to viscoelastic creep and, more importantly, due to the timedependency of adhesion (which is due to the velocity dependency of closing crack propagation). We have shown that the increase in the contact area manifests itself as an increase in the work of adhesion during pull-off.

(c) For rubber in contact with glass in water, we observe a very strong increase in the adhesion with increasing contact time, which we interpret as due to (thermally activated) dewetting transitions in the rubber-glass asperity contact regions.

(d) For the adhesion between the rubber and siliconised glass in silicone oil, we observe a very small work of adhesion, which is nearly independent of the contact time. This shows the stable and inert nature of the layer of silicone oil molecules chemically attached to the glass surface.

(e) For the adhesion between an (originally) clean glass surface and the rubber in silicone oil, we observe a slow decrease in the adhesion with the time period in which the glass was kept in the oil before the adhesion test. This indicates that silicone oil molecules, even at room temperature, slowly react with the glass surface to form a protective layer.

(f) We find that in silicone oil the pull-off force depends on the oil viscosity, while for water this viscous contribution to the pull-off force is negligible for the pull-off velocities used in our study. We have developed a simple theory which describes the influence of the fluid viscosity on the pull-off force.

\section{ACKNOWLEDGMENTS}

We thank Fabian Voll and Michael Stroh (Sanofi-Aventis Deutschland) for preparing the siliconisated glass balls. We thank K. Adamiak (West Pharmaceutical Services Deutschland) for supplying the rubber compound. This work was performed within a Reinhart-Koselleck project funded by the Deutsche Forschungsgemeinschaft (DFG). We would like to thank DFG for the project support under the reference German Research Foundation DFG-Grant No. MU 1225/36-1. This work is supported in part by the Research Council of Norway (Project No. 234115 in the Petromaks2 programme). 


\section{APPENDIX A: VISCOELASTIC MODULUS}

In this section, we present the results for the viscoelastic properties for the filled bromobutyl rubber compound used in the adhesion studies. We used a Q800 Dynamic Mechanical Analysis (DMA) instrument produced by TA Instruments. The machine is run in tension mode, meaning that a rectangular strip of rubber clamped on both sides, is elongated in an oscillatory manner. The complex viscoelastic modulus is first measured in constant strain mode with a strain amplitude of $0.04 \%$ and at different frequencies starting from $28 \mathrm{~Hz}$ and changed in steps until $0.25 \mathrm{~Hz}$ is reached (10 frequency points: 28.0, 25.0, 14.0, 7.9, 4.4, 2.5, 1.4, 0.79, 0.44, and $0.25 \mathrm{~Hz}$ ). The rather small strain amplitude is chosen in order to avoid strain softening effects, e.g., the Mullins effect ${ }^{55}$ or the Payne effect, ${ }^{56}$ which can strongly change the viscoelastic response of the rubber specimen. It is not clear how these nonlinear effects would affect the results at different temperatures, and one therefore usually measures the low strain master curve in the linear response region. From our previous experience, we have found that a strain amplitude of $0.04 \%$ is reasonably good for most rubber compounds. Measuring the rubber sample in tension mode also requires to prestrain the rubber with a static strain that has to be larger than the dynamic strain during oscillation. The prestrain in the experiments has been set to $0.06 \%$ to avoid compressing the rubber during the DMA measurement.

The experiment started at $-80^{\circ} \mathrm{C}$ and after measuring the modulus at all frequencies mentioned above, the temperature was increased in steps by $5^{\circ} \mathrm{C}$, and the procedure was repeated until $120{ }^{\circ} \mathrm{C}$ is reached. Note that it might be necessary to
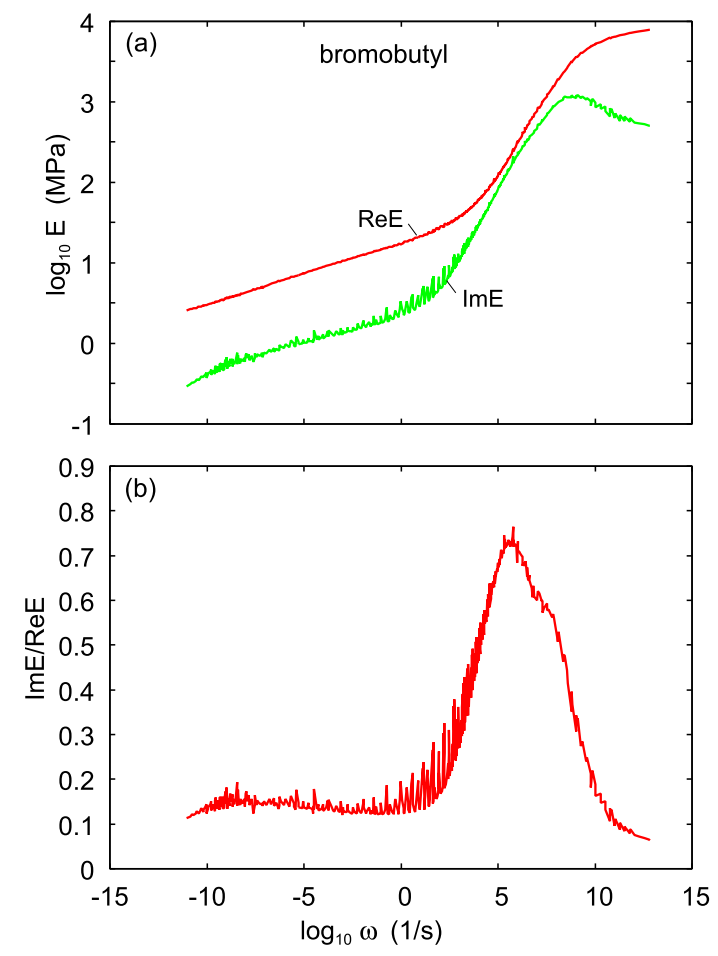

FIG. 27. The (a) real part $\operatorname{Re} E$ and (b) the imaginary part $\operatorname{Im} E$ of the rubber viscoelastic modulus, and the ratio $\operatorname{Im} E / \operatorname{Re} E$ as a function of the frequency $\omega$ experimentally measured at $0.04 \%$ strain amplitude and combined in the form of master curves for the reference temperature $T_{\text {ref }}=20^{\circ} \mathrm{C}$.

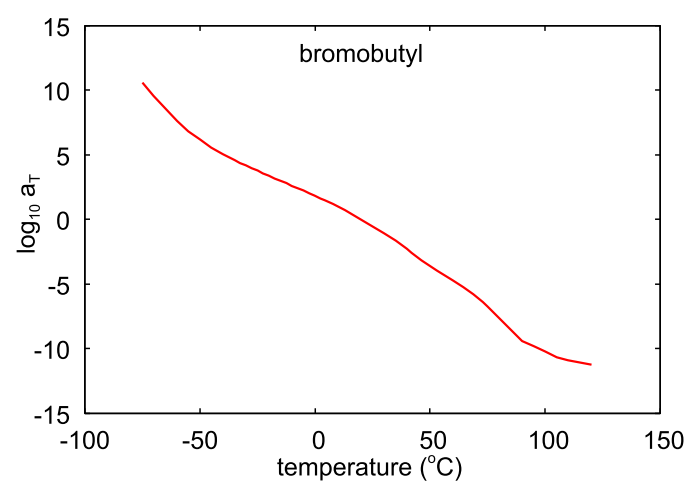

FIG. 28. The horizontal shift factors, $a_{T}$, as a function of the temperature after the measurements performed at $0.04 \%$ strain amplitude and the shift factors for the reference temperature $T_{\text {ref }}=20{ }^{\circ} \mathrm{C}$.

choose smaller temperature steps when reaching the glass transition temperature $T_{\mathrm{g}}$ where the viscoelastic response of the rubber material changes strongly with frequency (and temperature). This makes sure that the curves measured at different temperatures overlap with each other, which is necessary for the shift procedure to be accurate. The results are then shifted in order to form a smooth $\operatorname{Re} E$ master curve.

Figure 27 shows the (a) real part $\operatorname{Re} E$ and the imaginary part $\operatorname{Im} E$ of the rubber viscoelastic modulus, and (b) the ratio $\operatorname{Im} E / \operatorname{Re} E$ as a function of the frequency $\omega$. The measurements were performed at $0.04 \%$ strain amplitude and the master curves refer to the temperature $T_{\text {ref }}=20^{\circ} \mathrm{C}$. Figure 28 shows the horizontal shift factors, $a_{T}$, as a function of the temperature.

If we define the glass transition temperature $T_{\mathrm{g}}$ as the temperature where $\tan \delta=\operatorname{Im} E / \operatorname{Re} E$ as a function of temperature is maximal (for the frequency $\omega=0.01 \mathrm{~s}^{-1}$ ), then we obtain $T_{\mathrm{g}}=-62{ }^{\circ} \mathrm{C}$.

In the asperity contact regions between the rubber and the glass ball, the strain is usually very high, up to $\sim 1$ (where the strain $\epsilon=1$ corresponds to $100 \%$ strain). To take into account this, we have performed strain sweep up to very large strain $\epsilon \approx 1$. Figure 29 shows the ratio $\operatorname{Im} E(\epsilon) / \operatorname{Im} E(0)$ and $\operatorname{Re} E(\epsilon) / \operatorname{Re} E(0)$ as a function of the strain. The curves shown are polynomial fit curves to the average over measurements performed at $T=-20{ }^{\circ} \mathrm{C}, 0{ }^{\circ} \mathrm{C}$, and $20^{\circ} \mathrm{C}$.

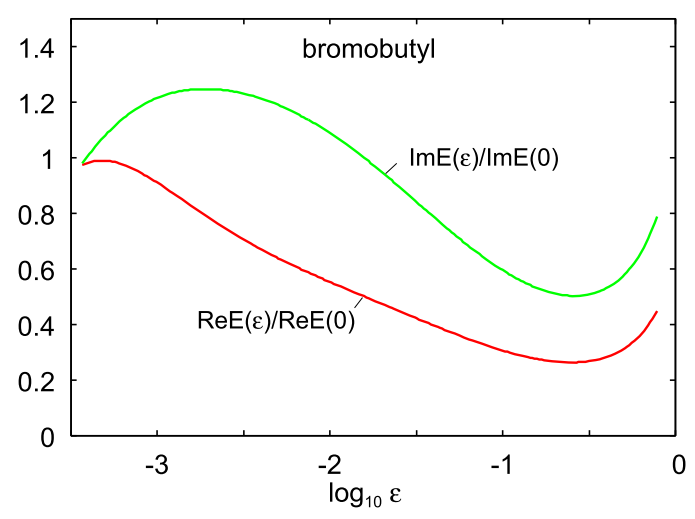

FIG. 29. The ratio $\operatorname{Im} E(\epsilon) / \operatorname{Im} E(0)$ and $\operatorname{Re} E(\epsilon) / \operatorname{Re} E(0)$ as functions of the strain (where the strain $\epsilon=1$ corresponds to $100 \%$ strain). The curves shown are polynomial fit curves to the average over the measurements performed at $T=-20{ }^{\circ} \mathrm{C}, 0^{\circ} \mathrm{C}$, and $20^{\circ} \mathrm{C}$. 


\section{APPENDIX B: CONTACT ANGLES AND SURFACE ENERGIES}

We have measured the advancing $\theta_{\mathrm{A}}$ and receding $\theta_{\mathrm{R}}$ contact angles for water and silicone oil on the glass ball surface and on the surface of the bromobutyl rubber and estimated the adiabatic work of adhesion between the rubber and the glass surface, in the dry state and in water and in silicone oil.

A small droplet of silicon oil deposited on the rubber or glass surface very quickly spreads out into a pancake like structure the diameter of which increased with time. Thus, $\theta_{\mathrm{A}}=0$ (complete wetting) for silicon oil on both the glass and the rubber surface. It follows that the thermal equilibrium contact angle $\theta_{\mathrm{c}}=0$.

For water, the measurements were performed by depositing a small water droplet on the rubber or glass surface (see Fig. 30). The advancing contact angle was determined after waiting $\sim 1 \mathrm{~min}$ at which point no movement of the contact line could be detected. The receding contact angle was determined by studying the water droplet during evaporation of the water. When enough water had evaporated the contact line started to move inwards and at this point we measured the receding contact angle. We performed 4 different experiments for (a) a glass ball cleaned with acetone, (b) the bromobutyl rubber surface cleaned with acetone, (c) a contaminated glass ball surface, which was first cleaned with acetone and then squeezed in contact (about 10 times) with the rubber, and (d) the same as (c) but with the rubber-glass contact in water. The
TABLE I. The water contact angle (in degree) on (a) a glass ball cleaned with acetone, (b) a bromobutyl rubber surface cleaned with acetone, (c) a contaminated glass ball surface, which was first cleaned with acetone and then ( 10 times) squeezed in contact with the rubber, and (d) the same as (c) but with the rubber-glass squeezed contact in water. We give the advancing $\theta_{\mathrm{A}}$, retracting $\theta_{\mathrm{R}}$, and (calculated) thermal equilibrium contact angle $\theta_{\mathrm{c}}$.

\begin{tabular}{lcrr}
\hline \hline system & $\theta_{\mathrm{A}}$ & $\theta_{\mathrm{R}}$ & $\theta_{\mathrm{c}}$ \\
\hline (a) Glass & 26.8 & 3.0 & 15.3 \\
(b) Rubber & 92.5 & 26.5 & 55.9 \\
(c) Contaminated glass & 56.0 & 3.5 & 28.5 \\
(d) Contaminated glass & 33.0 & 4.0 & 19.0 \\
\hline
\end{tabular}

measured advancing $\theta_{\mathrm{A}}$ and retracting $\theta_{\mathrm{R}}$ contact angles are given in Table I.

To estimate the thermal equilibrium contact angle $\theta_{\mathrm{c}}$, we used the expression proposed by Tadmor $^{58}$ (see also Chibowski ${ }^{59}$ )

$$
\theta_{\mathrm{c}}=\arccos \left(\frac{r_{\mathrm{A}} \cos \theta_{\mathrm{A}}+r_{\mathrm{R}} \cos \theta_{\mathrm{R}}}{r_{\mathrm{A}}+r_{\mathrm{R}}}\right),
$$

where

$$
\begin{aligned}
& r_{\mathrm{A}}=\left(\frac{\sin ^{3} \theta_{\mathrm{A}}}{2-3 \cos \theta_{\mathrm{A}}+\cos ^{3} \theta_{\mathrm{A}}}\right)^{1 / 3}, \\
& r_{\mathrm{R}}=\left(\frac{\sin ^{3} \theta_{\mathrm{R}}}{2-3 \cos \theta_{\mathrm{R}}+\cos ^{3} \theta_{\mathrm{R}}}\right)^{1 / 3} .
\end{aligned}
$$

The calculated thermal equilibrium contact angle $\theta_{\mathrm{c}}$ is also given in Table I.
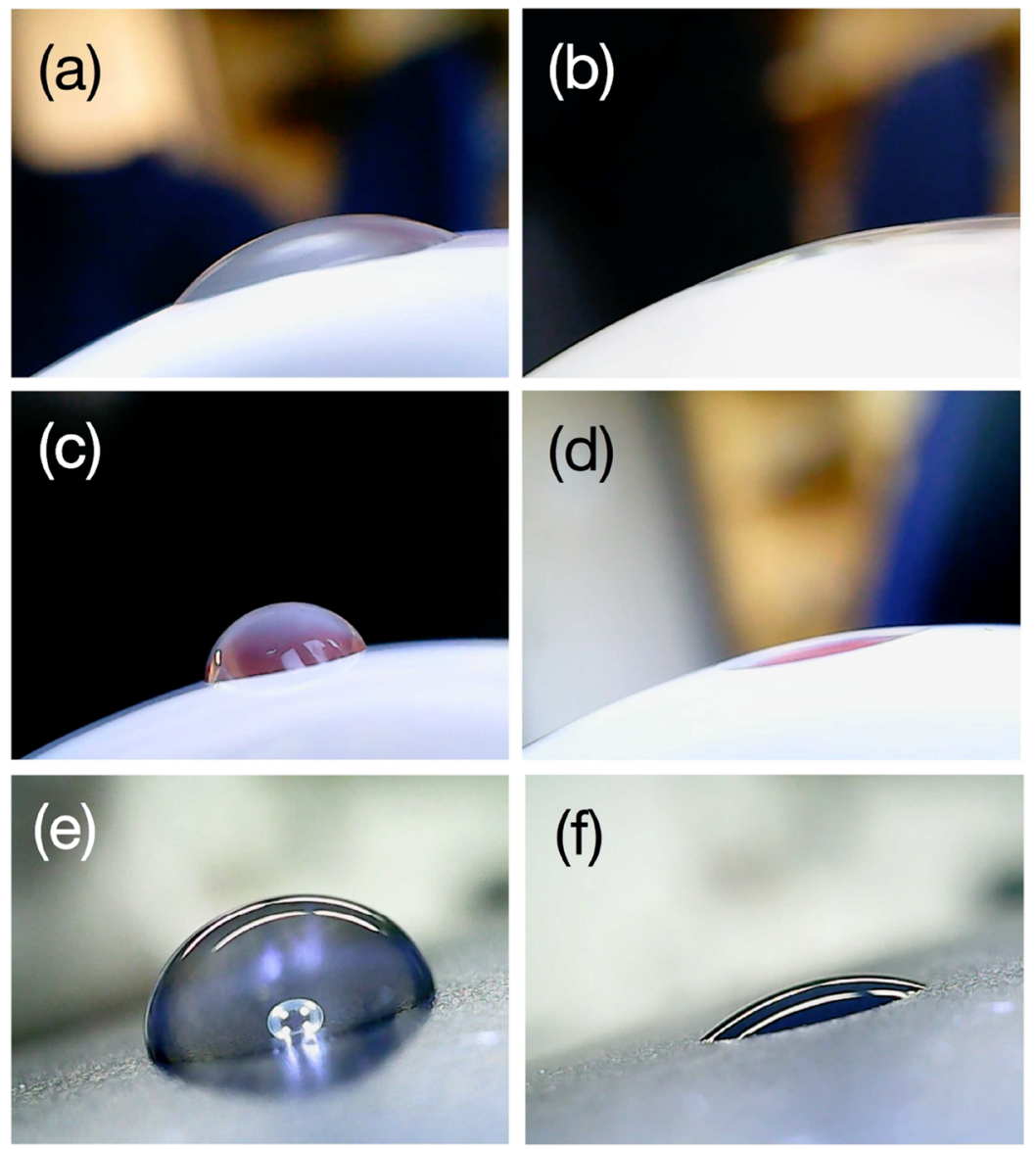

FIG. 30. Water droplet on the glass cleaned with acetone [(a) and (b)], on the glass contaminated by squeezing it in contact with the rubber [(c) and (d)], and on the rubber cleaned with acetone [(e) and (f)]. The left column [(a), (c), and (e)] is about $1 \mathrm{~min}$ after depositing the water droplet that is used to define the advancing contact angle. The right column [(b), (d), and (f)] is after most of the water has evaporated for defining the receding contact angle. For the movies of the time evolution of the water droplets, see Ref. 57. 
Note that after contact with the rubber the water contact angle on the glass ball has increased. We interpret this as resulting from transfer of uncrosslinked molecules from the rubber to the glass ball. When the contact between the rubber and the glass surface occurred in water, the change in the water contact angle is much smaller. We conclude that less molecules are transferred to the glass surface when the rubber-glass contact occurs in water as compared to that of the dry state. As will be shown in Sec. VI, the transfer of molecules to the glass surface when in contact with the rubber results in an adhesion force between the two solids which decreases with increasing number of contact cycles.

We also performed additional measurements of the water advancing contact angles on another (nominally identical) glass ball. The ball was first cleaned with acetone. Next, the ball was squeezed in contact with the rubber which gave the water advancing contact angle $42^{\circ}$.

Let us estimate $\Delta \gamma$ for the contact between the bromobutyl rubber and the glass surface. The surface energy (per unit surface area) for glass cleaned with acetone which results in a surface still covered by water and some (strongly bounded) organic contamination, is typically ${ }^{60} \gamma_{1} \approx 0.06-0.07 \mathrm{~J} / \mathrm{m}^{2}$. The surface energy for bromobutyl rubber is ${ }^{61} \gamma_{2} \approx 0.025$ $-0.035 \mathrm{~J} / \mathrm{m}^{2}$. In a simple approach, if only dispersion forces are responsible for the interaction between the glass and the bromobutyl rubber, the adiabatic work of adhesion is approximately given by ${ }^{62} \Delta \gamma \approx 2\left(\gamma_{1} \gamma_{2}\right)^{1 / 2}$, which in the present case gives $\Delta \gamma($ dry $) \approx 0.08-0.1 \mathrm{~J} / \mathrm{m}^{2}$. However, the experimental adhesion study presented below indicates that stronger bonds form between the two surfaces and $\Delta \gamma \approx 0.3 \mathrm{~J} / \mathrm{m}^{2}$.

In a liquid, the adiabatic work of adhesion can be calculated using the Young-Dupre equation (1). The liquid surface tension $\gamma \approx 0.072 \mathrm{~J} / \mathrm{m}^{2}$ for water and for silicone oil $\gamma \approx 0.02$ $\mathrm{J} / \mathrm{m}^{2}$.

Let us now estimate the adiabatic work of adhesion between bromobutyl rubber and the glass surface in water. As shown above, the adiabatic contact angle between water and the glass ball (cleaned by acetone) and the bromobutyl rubber (also cleaned by acetone) is $\theta_{\mathrm{gl}} \approx 15^{\circ}$ and $\theta_{\mathrm{rl}} \approx 56^{\circ}$, respectively. Assuming first only dispersion interaction $\Delta \gamma($ dry $) \approx 2\left(\gamma_{1} \gamma_{2}\right)^{1 / 2} \approx 0.09 \mathrm{~J} / \mathrm{m}^{2}$ from (1), we get $\Delta \gamma($ wet $)$ $\approx-0.02 \mathrm{~J} / \mathrm{m}^{2}$. Hence in this case, we obtain that $\Delta \gamma$ (wet) is negative, which implies no adhesion but a short ranged repulsion. However, as stated above, the adhesion studies presented below (see Sec. VI) show that $\Delta \gamma($ dry $) \approx 0.3 \mathrm{~J} / \mathrm{m}^{2}$ giving $\Delta \gamma($ wet $) \approx 0.2 \mathrm{~J} / \mathrm{m}^{2}$.

The negative work of adhesion predicted above is due to the dispersion force (i.e., the van der Waals interaction) which acts between the surfaces (in a modified form) also when a thin (nanometer) water film exists between the surfaces. ${ }^{62,63}$ Taking this into account we expect the interaction potential between a flat glass surface and a flat rubber surface in water to take the form shown in Fig. 2 with a repulsive barrier before the strong attraction due to direct rubber-glass bonds. That is, at separation of order a few nanometers the interaction is only via dispersion forces and steric repulsion and is repulsive. At short separation, the interaction potential has a local minimum corresponding to direct contact between the rubber and the glass surface. In this state, bonding forces (of unknown nature) stronger than the dispersion forces occur between the surfaces.

For silicone oil, the adiabatic contact angles $\theta_{\mathrm{gl}} \approx 0^{\circ}$ on glass and $\theta_{\mathrm{rl}} \approx 0^{\circ}$ on rubber. When one (or both) of the contact angles vanishes, it is not possible to use (1) to estimate the work of adhesion in the liquid, but this equation can only be used to give an upper limit for the work of adhesion. Thus, using $\Delta \gamma($ dry $) \approx 0.3 \mathrm{~J} / \mathrm{m}^{2},(1)$ gives $\Delta \gamma$ (wet) $<0.25 \mathrm{~J} / \mathrm{m}^{2}$. The adhesion measurements to be presented in Sec. VI indicate an initially very weak adhesion in silicone oil and in water, which increases with increasing time of contact.

\section{APPENDIX C: RUBBER SURFACE POWER SPECTRUM}

We have studied the rubber surface topography using an optical method and atomic force microscopy (AFM). As shown in Fig. 31, the height probability distribution is nearly Gaussian. Figure 32 shows the surface roughness power spectrum as a function of the wavenumber on the logarithmic scale. The green lines are from optical data, and the red lines are from AFM data. The dashed line is a fit to the data, where the tilted line has a slope corresponding to the Hurst exponent $H=0.92$ (or fractal dimension $D_{\mathrm{f}}=2.08$ ). In the calculations below, we use the dashed line linearly (on the logarithmic scale)

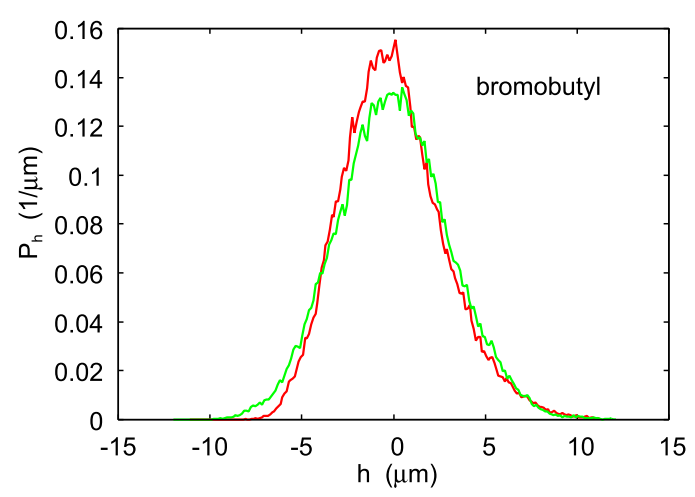

FIG. 31. The height probability distribution for the bromobutyl rubber surface as obtained from optical measured topography. The red and green lines are from two different surface areas.

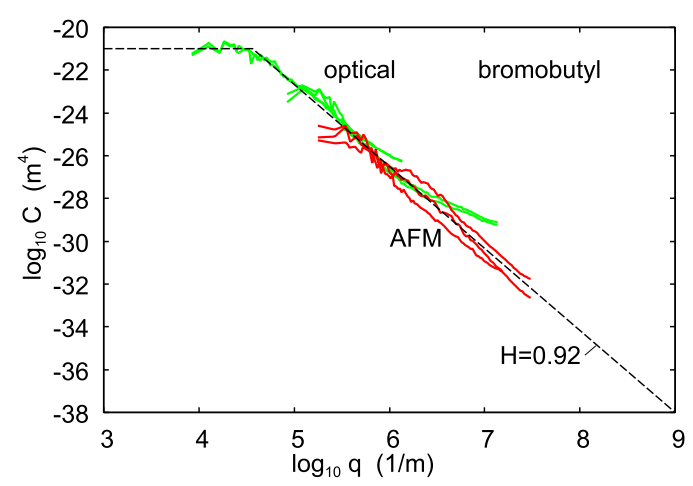

FIG. 32. The surface roughness power spectrum of the bromobutyl rubber surface as a function of the wavenumber (log-log scale). The green lines are from optical data, and the red lines are from atomic force microscopy (AFM) data. The dashed line is a fit to the data where the tilted line has a slope corresponding to the Hurst exponent $H=0.92$ (or fractal dimension $\left.D_{\mathrm{f}}=2.08\right)$. 
extrapolated to $q=q_{1}=10^{9} \mathrm{~m}^{-1}$. This power spectrum corresponds to a surface with the rms-slope 0.55 and rms-roughness $2.9 \mu \mathrm{m}$.

\section{APPENDIX D: INTERFACIAL CRACK PROPAGATION}

The contact line between a spherical probe and a rubber substrate can be considered as a crack tip and the work of adhesion equals the crack propagation energy per unit surface area $w$. It is well known that the crack propagation energy depends on the crack tip velocity $v$ and on the temperature $T$, i.e., $w=w(v, T)$. In addition it differs for a closing crack and an opening crack.

The crack propagation energy for an opening crack is often written as ${ }^{31,64-67}$

$$
\gamma_{\text {open }}(v, T)=\Delta \gamma[1+f(v, T)] .
$$

Here we are interested in interfacial (between the glass ball and the rubber substrate) crack propagation. In this case, as the crack velocity $v_{r} \rightarrow 0$ (when viscous effects in the rubber are negligible), the measured value of $\Delta \gamma$ can be identified as the energy $\Delta \gamma=\gamma_{1}+\gamma_{2}-\gamma_{12}$ needed to break the interfacial rubber-substrate bonds, which are usually of the van der Waals type.

For simple hydrocarbon elastomers, the effect of temperature can be completely accounted for by applying a simple multiplying factor, denoted by $a_{T}$, to the crack velocity $v$, i.e., $f(v, T)=f\left(a_{T} v\right)$. Moreover, values of $a_{T}$ found experimentally are equal to the Williams-Landel-Ferry (WLF) ${ }^{68}$ function determined from the temperature dependence of the bulk viscoelastic modulus. This clearly proves that the large effects of crack velocity and temperature on crack propagation in rubber materials are due to viscoelastic processes in the bulk.

In (D1), the function $f(v, T)=f\left(a_{T} v\right)$ describes the bulk viscoelastic energy dissipation in front of the crack tip. This term is determined by the viscoelastic modulus $E(\omega)$ of the rubber and can be calculated theoretically. The factor $\Delta \gamma$ is due to the bond breaking (in our applications between the rubber and the substrate) at the crack tip (in the so-called crack-tip process zone), which may involve highly non-linear processes. This term cannot be easily calculated theoretically and must be deduced directly from experimental data.

In Refs. 31 and 32, we have shown that

$$
\gamma_{\mathrm{open}}(v)=\Delta \gamma\left[1-\frac{2}{\pi} E_{0} \int_{0}^{2 \pi v / a} d \omega \frac{F(\omega)}{\omega} \operatorname{Im} \frac{1}{E(\omega)}\right]^{-1},
$$

where $E_{0}=E(0)$ and where

$$
F(\omega)=\left[1-\left(\frac{\omega a}{2 \pi v}\right)^{2}\right]^{1 / 2} .
$$

The crack tip radius $a=a(v)$ depends on the crack-tip velocity $v$ (and temperature) and can be determined if one assumes that the stress at the crack tip takes some critical value $\sigma_{\mathrm{c}}$. This gives

$$
\frac{a}{a_{0}}=\frac{\gamma_{\mathrm{open}}}{\Delta \gamma},
$$

where $a_{0}$ is the crack-tip radius for a very slowly moving crack. Note that the critical stress $\sigma_{\mathrm{c}}$ only enters in the adiabatic work of adhesion $w_{0}=2 \pi \sigma_{\mathrm{c}}^{2} a_{0} / E(0)$ and is therefore not an independent parameter. For high crack tip velocities, $\gamma_{\text {open }}(v)$ $\approx \Delta \gamma E(\infty) / E(0) \gg \Delta \gamma$. This is possible only if the denominator in the integral in (D2) is close to zero for high crack tip velocities, which means that the term involving the integral must be close to unity. If (D2) is used directly to calculate $\gamma_{\text {open }}(v)$ numerically, this requires that $E(\omega)$ is accurately known for all frequencies, which is usually not the case. However, it is possible to rewrite (D2) in a form convenient for numerical calculations. ${ }^{32}$ The predictions of the crack propagation theory presented above were compared to experimental data in Refs. 31, 32, 69, and 70. Note that for rubber materials $E(\infty) / E(0)$ is typically $\sim 1000$, so factor $f(v, T)$ in (D1) may enhance $w$ by a factor of $\sim 1000$ (or more) at high crack tip velocities.

\section{APPENDIX E: SHORT REVIEW OF THE JKR THEORY}

The contact region between a spherical probe (radius $R$ ) and a flat rubber surface is circular with the radius $r$. In the JKR theory, the interaction between the solids is described by the work of adhesion $w$, which is the energy per unit surface area to separate two flat surfaces from their equilibrium contact position to infinite separation. According to the JKR theory, the relation between the interaction force $F$ and the radius $r$ on the stable branch of the interaction curve is ${ }^{15,16}$

$$
r^{3}=\frac{3 R F_{\mathrm{c}}}{4 E^{*}}\left[\frac{F}{F_{\mathrm{c}}}+2+2\left(\frac{F}{F_{\mathrm{c}}}+1\right)^{1 / 2}\right],
$$

where $E^{*}=E /\left(1-v^{2}\right)$ (where $E$ and $v$ are the rubber Young's modulus and Poisson ratio, respectively), and where

$$
F_{\mathrm{c}}=\frac{3 \pi}{2} w R
$$

is the pull-off force. Thus for an elastic solid, if the ball is pulled by a soft spring (and neglecting inertia effects), at $F=-F_{\mathrm{c}}$ the pull-off force abruptly drops to zero.

It is well-known that the separation line $r=r(t)$ can be considered as a crack tip. ${ }^{67,71}$ The work of adhesion $\gamma_{\text {open }}$ in general depends on the velocity $v_{r}=\dot{r}$ of the opening (during pull-off) or closing (during contact formation) crack tip. At finite crack tip velocity, for an opening crack, $\gamma_{\text {open }}$ can be strongly enhanced (and for a closing crack $\gamma_{\text {close }}$ strongly reduced), compared to the adiabatic (infinitely low crack tip velocity) value $\Delta \gamma$. One contribution to the work of adhesion is derived from the viscoelastic energy dissipation in the vicinity of the crack tip [see Fig. 33(a)]. For an opening crack, this will enhance $w$ with a factor of $1+f\left(v_{r}, T\right)$, which depends on the crack tip velocity $v_{r}$ and the temperature $T$. For a closing crack, the corresponding reduction factor is approximately ${ }^{72}$ $\approx 1 /\left[1+f\left(v_{r}, T\right)\right]$.

Since the work of adhesion depends on the crack tip velocity $v_{r}=\dot{r}(t)$ we need to determine this quantity. In the experiments, we calculate $v_{r}$ from the time dependency of $F(t)$ assuming that the JKR theory is valid. Thus using (E1), we can obtain $r(t)$ from the measured $F(t)$. During pull-off, the velocity $v_{r}$ varies with time, but what is most important is the velocity at the point when the pull-off force is maximal; this is the crack tip velocity quoted below. 
(a)

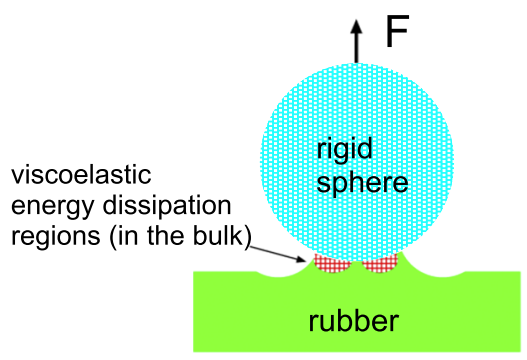

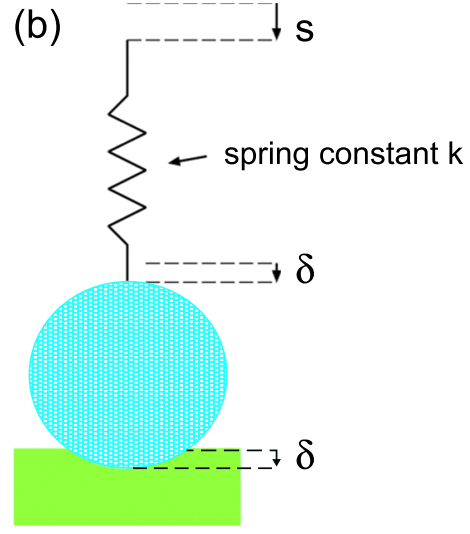

FIG. 33. (a) A rigid ball pulled away from a viscoelastic solid. A part of the energy needed to remove the ball is derived from the viscoelastic energy dissipation inside the rubber close to the opening crack tip (the red dashed region). (b) If the spherical body can be treated as rigid, the penetration $\delta$ is equal to the displacement of the uppermost point of the ball toward the substrate where $\delta=0$ corresponds to the case when the ball just touches the substrate in one point. Often $\delta$ is not measured directly but rather the displacement $s$ further away and in this case some elastic element (spring constant $k$ ) relates the displacements $\delta$ and $s$ via $k(s-\delta)=F$, where $F$ is the force exerted by the ball on the substrate.
In two earlier publications ${ }^{45,49}$ we have, for rubber materials, tested the JKR theory predictions above using another set-up where $r(t)$ was measured directly using an optical microscopy. We found good agreement between the experiments and the JKR theory prediction. Thus, in analyzing the experiments presented below, we have obtained $v_{r}$ from the time dependency of the interaction force $F(t)$ using (E1).

\section{APPENDIX F: INTERFACIAL SEPARATION WITHOUT ADHESION}

Contact mechanics in the presence of interfacial fluid is a complex problem, ${ }^{44}$ and here we only employ a simple model for how the interfacial separation vary with time when the loading force is reduced from its maximum value to zero for the ball-rubber contact in silicone oil. The pull-off during the time period when $F(t)<0$ will be studied in Appendixes $\mathrm{H}$ and I. We assume first that there is no adhesion at the interface. Let $t=0$ correspond to the time when we start to pull-off the ball and assume the interaction force $F(t)=\left(1-t / t_{0}\right) F_{0}$, where $F_{0}$ is the maximum of the loading force, usually on order $0.1 \mathrm{~N}$ and $t_{0}$ is the time necessary to reduce the force to zero, which depends on the pull-off velocity $v_{z}$, but which is typically $100-1000 \mathrm{~s}$. As shown in Sec. III during the loading phase [see the green curve in Fig. 6(a)] for loading times larger than $10 \mathrm{~s}$, the average interfacial separation is not influenced by the fluid but is determined by the interaction between the glass surface and the rubber asperities. Thus we will assume that at $t=0$ (start of pull-off) the (average) interfacial separation $u=u_{0}$ takes the value expected for static loading without the fluid. We will now study how $u(t)$ increases as the force $F(t)$ decreases linearly toward zero assuming that $u(t=0)=u_{0}$.

When the loading force is maximal, the Hertz contact between the glass ball and the rubber surface is circular with radius $r_{0}$ and the average contact pressure $p_{0}=F_{0} /\left(\pi r_{0}^{2}\right)$. We now consider a simplified situation where instead of removing a ball we remove a circular disc with radius $r_{0}$ from the rubber surface. We will also neglect the influence of the surface roughness on the fluid flow dynamics. In the present case, the average contact pressure is below $0.1 \mathrm{MPa}$ and the fluid pressure flow factor, which determines the influence of the surface roughness on the fluid flow, is close to unity so neglecting the influence of the surface roughness on the fluid flow is a good approximation.
The initial contact pressure $p_{0}$ is so small that we can use the asymptotic relation $p(u)$ for the rubber-glass interaction:

$$
p=\beta E^{*} e^{-u / u_{a}} .
$$

Thus the initial separation

$$
u_{0}=-u_{a} \log \left(\frac{p_{0}}{\beta E^{*}}\right) .
$$

The parameters $u_{a}$ and $\beta$ can be calculated from the surface roughness power spectrum and we get $\beta=0.069$ and $u_{a}=1.45$ $\mu \mathrm{m}$. Neglecting adhesion the loading force $F(t)$ must equal the asperity contact force $\pi r^{2} p$ plus the viscous force, i.e.,

$$
F(t)=-\frac{3 \pi}{2} \eta r_{0}^{4} \frac{\dot{u}}{u^{3}}+\pi r_{0}^{2} \beta E^{*} e^{-u / u_{a}} .
$$

Assuming $F(t)=F_{0}\left(1-t / t_{0}\right)$, one can solve (F1) numerically for $u(t)$. In Fig. 34, we show the results for the disc removed from the elastic substrate (with Young's modulus $E=2.5 \mathrm{MPa}$ ) in a fluid with the viscosity $\eta=0.35 \mathrm{~Pa}$. The disc has the radius $r_{0} \approx 0.655 \mathrm{~mm}$, and the substrate has random roughness with the power spectrum given in Fig. 32. The squeezing force on the disc decreases linearly with time from $F=F_{0}=0.1 \mathrm{~N}$ at time $t=0$ to $F=0$ at time $t=t_{0}$. The average surface separation $u(t)$ is shown as a function of time for the case $t_{0}=100 \mathrm{~s}$ (the green line) and $t_{0}=1000 \mathrm{~s}$ (the red line). Note that the average surface separation at $t=t_{0}$ is $u\left(t_{0}\right)=8.8$

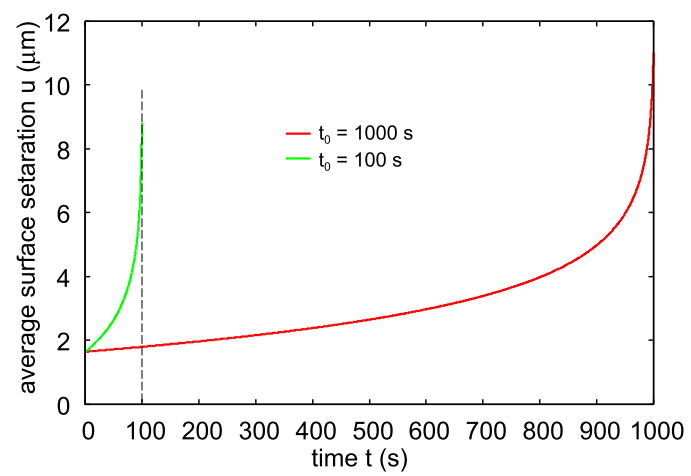

FIG. 34. A circular disc removed from an elastic substrate in a fluid with the viscosity $\eta=0.35 \mathrm{~Pa}$ s. The disc has the radius $r_{0} \approx 0.655 \mathrm{~mm}$ and the substrate has random roughness with the power spectrum given in Fig. 34. The squeezing force on the disc decreases linearly with time from $F=F_{0}$ $=0.1 \mathrm{~N}$ at time $t=0$ to $F=0$ at time $t=t_{0}$. The average surface separation $u(t)$ is shown as a function of time for the case $t_{0}=100 \mathrm{~s}$ (the green line) and $t_{0}=1000 \mathrm{~s}$ (the red line). The rubber Young's modulus $E=2.5 \mathrm{MPa}$ and the average contact pressure at $t=0$ is $p_{0}=F_{0} /\left(\pi r_{0}^{2}\right)=0.074 \mathrm{MPa}$. 
and $11.0 \mu \mathrm{m}$, respectively. However, in order to explain the observed pull-off forces, one needs to assume $u\left(t_{0}\right)=1.3-1.8$ $\mu \mathrm{m}$ (see Sec. VI and Appendix H). This indicates that due to adhesion the average surface separation at $t=t_{0}$ is almost the same as when the loading force is maximal $[u(t=0) \approx 1.65$ $\mu \mathrm{m}$ according to Fig. 34]. We attribute this to strong adhesive contact hysteresis.

For water, the increase in the interfacial separation during the unloading process, when $F$ changes from its maximum to zero, will be even larger than for the silicone oil due to its smaller viscosity. However, for the case of water the optical microscopy pictures of the rubber-glass contact do not show any change when the loading force is changed from its maximum to zero. This again indicates that the energy to propagate the opening crack (at relevant speeds) is so large that the compressed asperities cannot reduce the contact area during unloading. For contact in silicone oil, it is not possible to directly observe the contact area with our optical microscope because the contrast between contact and not contact is too low.

\section{APPENDIX G: ADHESION WITH SILICONE OIL FILMS ON THE RUBBER AND GLASS SURFACES}

We have studied adhesion when both the rubber and the glass ball were covered with thin films (thickness $d$ ) of silicone oil. The adhesion data are analyzed using the JKR theory although this theory may not be strictly valid in the present case. Hence, the "work of adhesion" quoted below must be interpreted simply as $w=2 F_{\mathrm{c}} /(3 \pi R)$. We note that a fluid capillary bridge (with fixed fluid volume) between a rigid sphere and a flat rigid surface gives rise to a (adiabatic) pulloff force $F_{\mathrm{c}}=4 \pi R \gamma$, where we have assumed that the fluid wet the two solids. ${ }^{62}$ Thus in this limit the "work of adhesion" must be interpreted as $w=8 \gamma / 3 \approx 2.67 \gamma$. We believe that this result holds accurately also when a fluid film exists at the interface if the fluid film thickness is large compared to the root-mean-square roughness amplitude. In fact, we have recently performed experiments where the substrate was a thick $(d \approx 1 \mathrm{~cm})$ silicone oil slab (the same oil as used here), and where $F_{\mathrm{c}}=4 \pi R \gamma$ was found to hold accurately. ${ }^{73}$

In the opposite limit where the surface roughness amplitude is much larger than the oil film thickness the JKR theory is approximately valid with $w=2 \gamma$ (see Ref. 74). For finite pull-off speeds, the pull-off force is also influenced by the fluid viscosity.

Figure 35 shows the "work of adhesion" during retraction (separation) (filled circles) and during approach (open circles), as a function of the number of contacts between the glass ball and the rubber surface. The glass ball and rubber surface are covered by $\sim 10 \mu \mathrm{m}$ film of silicone oil (viscosity $\approx 0.35 \mathrm{~Pa} \mathrm{~s}$ ). The red and blue circles are the work of adhesion for the pull-off (and approach) velocities $0.9 \mu \mathrm{m} / \mathrm{s}$ and 35 $\mu \mathrm{m} / \mathrm{s}$, respectively.

In this case, the work of adhesion is determined by the silicone oil capillary bridge formed between the glass ball and the rubber surface. This results in an adhesive interaction force, which extends over much larger separation distance (or time) then for the dry state. This is illustrated in Fig. 36 which shows

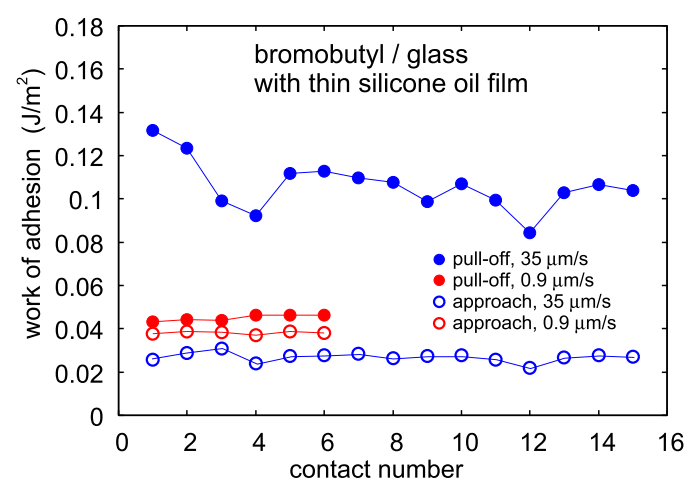

FIG. 35. The work of adhesion during retraction (separation) (filled circles) and during approach (open circles), as a function of the number of contacts for the rubber surface. The glass ball and rubber surface were originally cleaned with acetone and after covered with a thin film of silicone oil (viscosity $\approx 0.65 \mathrm{~Pa} \mathrm{~s}$ ). The red and blue circles are the work of adhesion for the pull-off or approach velocities $0.9 \mu \mathrm{m} / \mathrm{s}$ and $35 \mu \mathrm{m} / \mathrm{s}$, respectively.

the interaction force as a function of time close to a detachment for the drive (pull-off) speed $v_{z}=0.9 \mu \mathrm{m} / \mathrm{s}$. Note that $\sim 100 \mathrm{~s}$ (or the distance $\sim 100 \mu \mathrm{m}$ ) is necessary in order to break the junction, in contrast to the $\sim 1 \mathrm{~s}$ (or the distance $\sim 1 \mu \mathrm{m}$ ) for the dry surface [see Fig. 23(a) below].

In this study, we do not know the exact oil thickness but if the fluid film is thinner than the root-mean-square roughness amplitude, the adiabatic work of adhesion $w_{0}$ resulting from a capillary bridge is just twice the surface tension $\gamma$ of the liquid, $w_{0}=2 \gamma$ (see Ref. 74). The actual work of adhesion is affected by viscous dissipation in the liquid bridge and, to a much less extent, in the rubber, which will enhance $w$ during pull-off (say from $w_{0}$ to $w_{a}$ ) and reduce $w$ during approach (say from $w_{0}$ to $w_{b}$ ). Here we assume, as found to hold to a good approximation for the viscoelastic crack propagation case, that the enhancement factor $w_{a} / w_{0}=(1+f)$ during pull-off is also determining (approximately) the reduction factor $w_{b} / w_{0}=1 /(1$ $+f$ ) during approach. In this case $w_{0}=\left(w_{a} w_{b}\right)^{1 / 2}$.

The silicone oil we use has the surface tension $\gamma \approx 0.02$ $\mathrm{J} / \mathrm{m}^{2}$ so we get $w_{0}=2 \gamma \approx 0.04 \mathrm{~J} / \mathrm{m}^{2}$. From Fig. 35 , the work of adhesion at the lowest pull-off speed $0.9 \mu \mathrm{m} / \mathrm{s}$ is $w_{a} \approx 0.05 \mathrm{~J} / \mathrm{m}^{2}$ during pull-off and $w_{b} \approx 0.03 \mathrm{~J} / \mathrm{m}^{2}$ during approach. Assuming $w_{0} \approx\left(w_{a} w_{b}\right)^{1 / 2}$, we get the adiabatic work of adhesion $\approx 0.04$ $\mathrm{J} / \mathrm{m}^{2}$ in good agreement with the expected adiabatic work of adhesion.

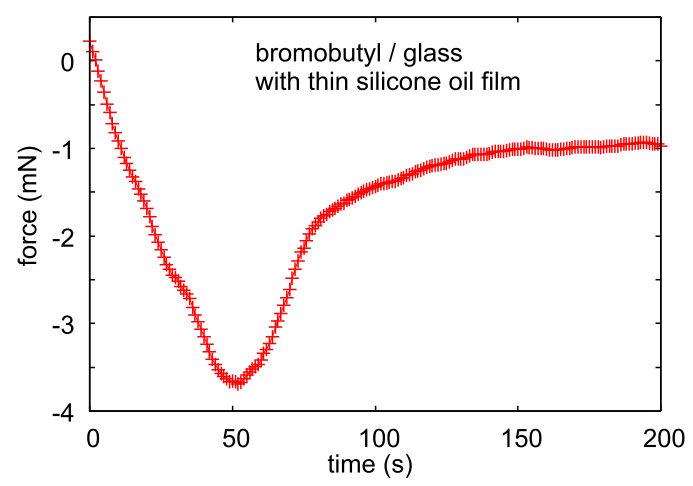

FIG. 36. The interaction force between the glass ball and the bromobutyl rubber as a function of time close to a detachment for surfaces covered by a thin film of silicone oil (viscosity $0.35 \mathrm{~Pa} \mathrm{~s}$ ). The drive speed is $v_{z}=0.9 \mu \mathrm{m} / \mathrm{s}$. 


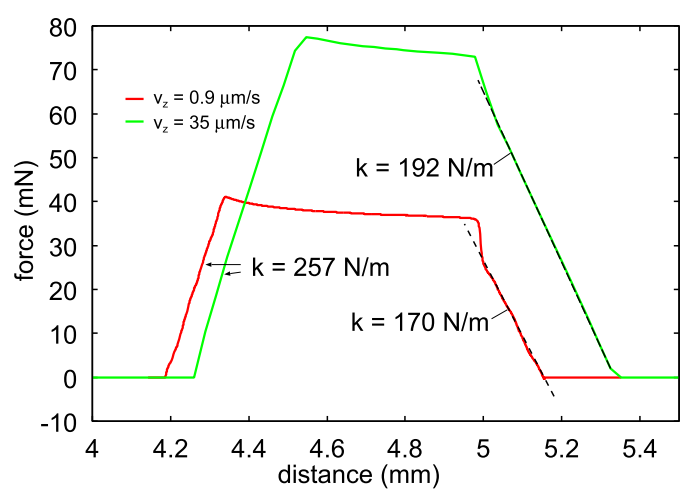

FIG. 37. The relation between the force and the displacement of the drive during a contact cycle of the glass ball against an approximately rigid (in the case PMMA) plate. The effective spring constant during retraction depends slightly on the retraction velocity but is of order $k \approx 200 \mathrm{~N} / \mathrm{m}$.

The work of adhesion at the higher pull-off speed (35 $\mu \mathrm{m} / \mathrm{s}$ ) is larger than for the lower speed due to the increased viscous dissipation. For $35 \mu \mathrm{m} / \mathrm{s}$ from Fig. 35 , we get $w_{a} \approx 0.13$ $\mathrm{J} / \mathrm{m}^{2}$ and $w_{b} \approx 0.015 \mathrm{~J} / \mathrm{m}^{2}$. Thus we get $\left(w_{a} w_{b}\right)^{1 / 2} \approx 0.04 \mathrm{~J} / \mathrm{m}^{2}$ again in agreement with the expected adiabatic work of adhesion. In Ref. 73, where we studied adhesion during (slow) cross-linking of silicone oil, we did not observe any effect of the silicone oil viscosity on the pull-off force until very close to the gel point of the silicone oil (where the viscosity becomes huge), but we attribute this to the different experimental conditions. In Ref. 73, the glass ball interacted with a bulk oil sample, while in the present study the silicon oil film is very thin, and the viscous energy dissipation is much larger in a thin fluid film (assuming similar flow velocities) than in a bulk sample.

\section{APPENDIX H: ROPE SPRING CONSTANT}

The nylon rope connecting the glass ball to the pulley can be considered as an effective spring with the spring constant $k$. We can measure $k$ by moving the glass ball toward and away from a hard substrate. Figure 37 shows the relation between the force and the displacement of the drive during a contact cycle of the glass ball against an approximately rigid (in the case PMMA) plate. The effective spring constant during retraction depends slightly on the retraction velocity but is of order $k \approx 170-190 \mathrm{~N} / \mathrm{m}$.

\section{APPENDIX I: FLUID SQUEEZE-IN, NUMERICAL RESULTS}

For adhesion in the silicone oil, the interaction force during retraction is strongly influenced by the fluid viscosity, which results in a much slower time variation in the interaction force at pull-off than the very fast (abrupt) (within $1 \mathrm{~s}$ ) variation observed in water. Here we present a very simple approximate theory for the influence of the silicone oil on the interaction force during pull-off.

Consider a glass ball squeezed against the rubber surface in a fluid. Let $t=0$ denote the time during pull-off when the force on the substrate vanishes (which was denoted by $t_{0}$ in Appendix F). Let $u_{0}$ be the average separation between the surfaces in the nominal contact area at time $t=0$. When (during unloading) the interacting force vanishes, there is a fluid pressure force at the interface and an equal (but of opposite sign) asperity contact pressure force. Here we will make the assumption that when $F=0$ the fluid pressure force vanishes (which implies that the repulsive and attractive rubber-glass asperity forces balance each other). To calculate the dependency of the interaction force $F(t)$ on time, we assume that for $t>0$ the only force acting on the ball is the viscous force from the fluid. Thus we assume that the attractive glass-rubber interaction matters only in that it determined the initial (average) surface separation $u(0)=u_{0}$ when $F=0$.

When a rigid ball (radius $R$ ) is squeezed against a flat rigid surface in a fluid with the viscosity $\eta$, the relation between the force $F$ and the (minimum) surface separation $u$ is given by ${ }^{75,76}$

$$
F=-6 \pi \eta R^{2} \frac{\dot{u}}{u} .
$$

As shown in Appendix $\mathrm{J}$, this equation can also be derived by considering the fluid squeeze-out for a circular plate with radius $r=(2 R u)^{1 / 2}$ (see Fig. 38).

The interfacial separation velocity $\dot{u}(t)$ is not the same as the drive velocity $v_{z}$ because of the elasticity in the system. Thus the nylon rope has an effective spring constant $k \approx 100$ $-200 \mathrm{~N} / \mathrm{m}$ (see Appendix H), and the rubber substrate too will deform elastically in response to the viscous force $F$ acting on it. As a result $F=k^{*}(s-u)$, where $s=s_{0}+v t$ is the drive displacement and $k^{*}<k$ is an effective (combined) spring constant (see Appendix J). Combining this equation with (I1) gives the equation of motion for $u(t)$ (here we have neglected inertia effects)

$$
6 \pi \eta R^{2} \frac{\dot{u}}{u}=k^{*}(s-u) .
$$

Let $t=0$ correspond to the point during retraction where the force on the substrate from the ball vanishes $(F=0)$. We assume that when $F \leq 0$ only the viscous force acts on the ball (and the substrate). Since $F=0$ at $t=0$ from (I1), we get that $\dot{u}=0$ for $t=0$. The second boundary condition $u(0)=u_{0}$ is determined by the separation between the glass ball and the rubber substrate at $t=0$ and will be used as a fitting parameter (the only fitting parameter) in the following study.

We have solved (I2) using numerical integration (see Appendix J). In Figs. 39, 40, and 23(c), we compare the theory predictions with the measured data for the pull-off speeds $v_{z}=35,6.3$, and $1.75 \mu \mathrm{m} / \mathrm{s}$. The results are for the bromobutyl

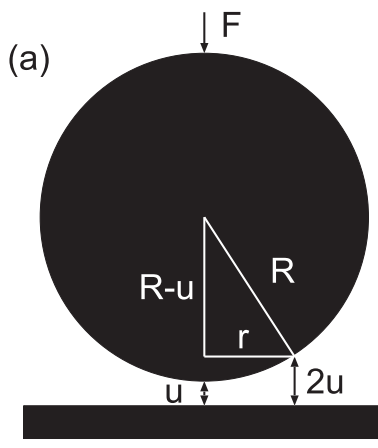

(b)

FIG. 38. The rate of fluid squeeze-out for the sphere with radius $R$ is the same as for a circular disc with radius $r=(2 R u)^{1 / 2}$. 


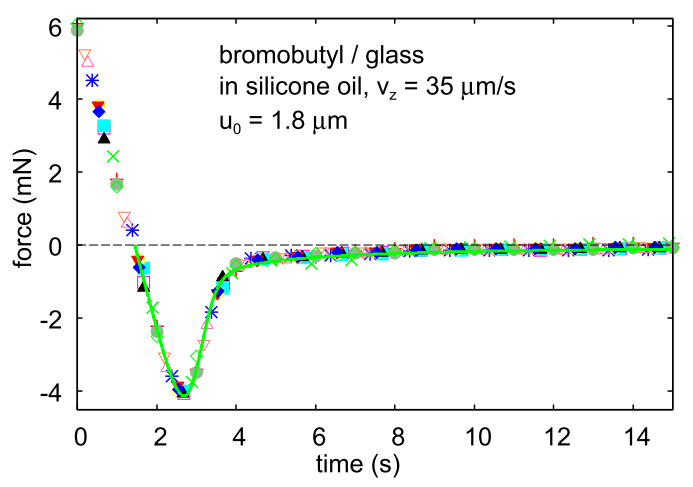

FIG. 39. The force $F(t)$ acting on the substrate during pull-off of a glass ball (diameter $2 R=3 \mathrm{~cm}$ ) in silicone oil. The pull-off speed $v_{z}=35 \mu \mathrm{m} / \mathrm{s}$. The different symbols are from different pull-off cycles, shifted along the time axis so that the minimum of the pull-off force occurs at the same time point. The green line is the theory prediction assuming that only a viscous drag force acts on the rubber substrate. For the bromobutyl rubber in silicone oil with the viscosity $\eta=0.35 \mathrm{~Pa}$ s. For the theory curve, we have assumed that the initial separation (at $F=0$ ) between the glass ball and the substrate is $u_{0}=1.8 \mu \mathrm{m}$.

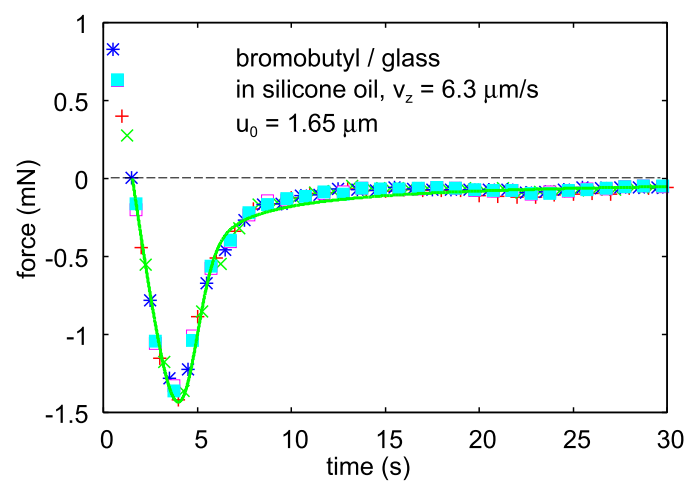

FIG. 40. The same as in Fig. 39 but for the pull-off speed $v_{z}=6.3 \mu \mathrm{m} / \mathrm{s}$. For the theory curve, we have assumed that the initial separation (at $F=0$ ) between the glass ball and the substrate is $u_{0}=1.65 \mu \mathrm{m}$.

rubber in silicone oil with the viscosity $\eta=0.35 \mathrm{~Pa}$ s. The figures show the force $F(t)$ acting on the substrate during pull-off of a glass ball (diameter $2 R=3 \mathrm{~cm}$ ) in silicone oil. The different symbols are from different pull-off cycles, shifted along the time axis so that the minimum of the pull-off force occurs at the same time point. The green lines are the theory prediction assuming that only a viscous drag force acts on the rubber

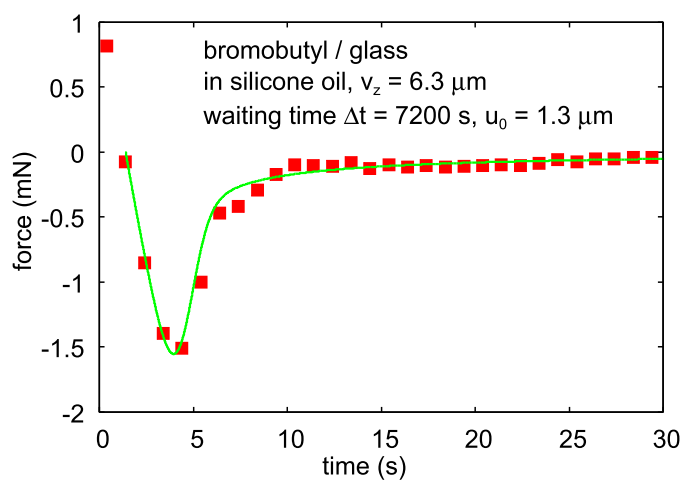

FIG. 41. The same as in Fig. 40 but for the waiting time 7200 s. We have assumed the initial separation (at $F=0$ ) $u_{0}=1.3 \mu \mathrm{m}$. substrate. The separation $u_{0}$ when $F=0$ is found to be $u_{0}=1.8$, 1.65 , and $1.4 \mu \mathrm{m}$. Note that $u_{0}$ decreases with decreasing (approach and pull-off) speed $v_{z}$. We interpret this as resulting from the longer glass-rubber contact time at the lower speed (the contact time is proportional to $1 / v_{z}$ ). As a further test, we performed another experiment with the pull-off velocity 6.3 $\mu \mathrm{m} / \mathrm{s}$ where we kept the ball in contact with the rubber for $7200 \mathrm{~s}$ before removing the contact. In this case, we found that using $u_{0}=1.3 \mu \mathrm{m}$ gives good agreement with the experiment, see Fig. 41. This is considerable smaller than $1.65 \mu \mathrm{m}$ found without the waiting time period. This is in accordance with the results obtained in Sec. VIE, where we found that for the clean glass ball the longer contact time results in a stronger adhesive interaction between the glass ball and the rubber, and hence to a smaller $u_{0}$.

\section{APPENDIX J: FLUID SQUEEZE-IN, THEORY}

Consider the fluid squeeze flow between a rigid sphere and a flat rigid surface. Let $u(t)$ denote the minimum separation between the surfaces. Most of the resistance toward fluid squeeze-out occurs in the region where the separation between the surfaces arises from the area where the separation between the surfaces is of order a few times $u(t)$, say when the separation varies from $u(t)$ to $2 u(t)$. When $u \ll R$, the radius of this circular region is $r \approx(2 R u)^{1 / 2}$ (see Fig. 38). In a first approximation, we can replace the sphere with a circular disc with radius $r$ separated from the flat substrate with the distance $u$. For a circular disk, the relation between the applied force $F$ and the separation $u$ is given by the well-known relation (see, e.g., Refs. 1 and 2)

$$
F=-\frac{3 \pi}{2} \eta r^{4} \frac{\dot{u}}{u^{3}}
$$

Substituting $r \approx(2 R u)^{1 / 2}$ in this equation gives

$$
F=-6 \pi \eta R^{2} \frac{\dot{u}}{u},
$$

which agrees with the exact result (I1).

Let us now study the fluid squeeze flow when the drive is moved away (pull-off) from the substrate with the speed $v$. We will neglect inertia effects. Thus, the same force $F$ which acts on the sphere from the driving spring also acts on the substrate. This will result in some elastic deformation of the substrate. Let $q$ denote the displacement of the substrate at the center of the contact. If a uniform stress act on a semi-infinite solid within a circular region with radius $r$, the displacement $q$ is related to the force $F$ via $F=k^{\prime} q$, where $k^{\prime}=(\pi / 2) r E^{*}$ [where $E^{*}=E /\left(1-v^{2}\right)$, where $E$ and $v$ are the Young's modulus and the Poisson ration of the rubber]. Using this equation and $F=k(s-w)$, where $s=s_{0}+v t$ is the displacement of the drive and $w$ is the displacement of the sphere and $k$ is the spring constant of the driving spring (see Appendix $\mathrm{H}$ ), we get the separation at the interface

$$
u=w-q=s-\left(\frac{1}{k}+\frac{1}{k^{\prime}}\right) F=s-\frac{1}{k^{*}} F .
$$

In the present study $k \ll k^{\prime}$ and the effective substrate (rubber) spring constant $k^{\prime}$ is not very important for the results presented below. 
Using (J1) this gives

$$
6 \pi \eta R^{2} \frac{\dot{u}}{u}=k^{*}(s-u)
$$

or

$$
\dot{u}=(s-u) u \alpha,
$$

where

$$
\alpha=\frac{k^{*}}{6 \pi \eta R^{2}} .
$$

Note that since $k^{\prime}=(\pi / 2) r E^{*}=(\pi / 2)(2 R u)^{1 / 2} E^{*}$ depends on $u$ so will $\alpha=\alpha(u)$. The solution of (J2) depends on the initial condition for $u(t)$ for $t=0$ and on $s_{0}$. These values depend on the history of the contact prior to the time $t=0$, which depends on the influence of the surface roughness on the contact between the ball and the rubber substrate.

Let us first consider a general case, where $u(0)=u_{0}$ and $\dot{u}(0)=v_{0}$. Writing

$$
u=v_{0} t+u_{0}+v \xi
$$

this gives $\xi=\dot{\xi}=0$ for $t=0$. Substituting (J4) in (J2) gives

$$
v_{0}+v \dot{\xi}=\left(s_{0}+v t-v_{0} t-u_{0}-v \xi\right) u \alpha .
$$

For $t=0$ from (J2) we get $v_{0}=\left(s_{0}-u_{0}\right) u_{0} \alpha_{0}$, where $\alpha_{0}=\alpha\left(u_{0}\right)$. Thus $s_{0}-u_{0}=v_{0} /\left(\alpha_{0} u_{0}\right)$. Substituting this in (J5) gives

$$
\dot{\xi}=-\frac{v_{0}}{v}+\left[\frac{v_{0}}{v \alpha_{0} u_{0}}+\left(1-\frac{v_{0}}{v}\right) t-\xi\right] u \alpha(u),
$$

where

$$
\alpha=\frac{k}{6 \pi \eta R^{2}}\left(1+\frac{k}{k_{0}^{\prime}}\left(\frac{u_{0}}{u}\right)^{1 / 2}\right)^{-1},
$$

with $k_{0}^{\prime}=(\pi / 2)\left(2 R u_{0}\right)^{1 / 2} E^{*}$.

Let $t=0$ correspond to the point during retraction where the force on the substrate from the ball vanishes $(F=0)$. We assume that when $F \leq 0$ only the viscous force acts on the ball (and the substrate). Since $F=0$ at $t=0$ from (J1), we get that $\dot{u}=0$ at $t=0$. Thus $v_{0}=0$ and (J6) reduces to

$$
\dot{\xi}=(t-\xi)\left(u_{0}+v \xi\right) \alpha\left(u_{0}+v \xi\right) .
$$

We have solved (J8) using numerical integration. In Figs. 39, 40, and 23(c), we show the force $F(t)$ acting on the

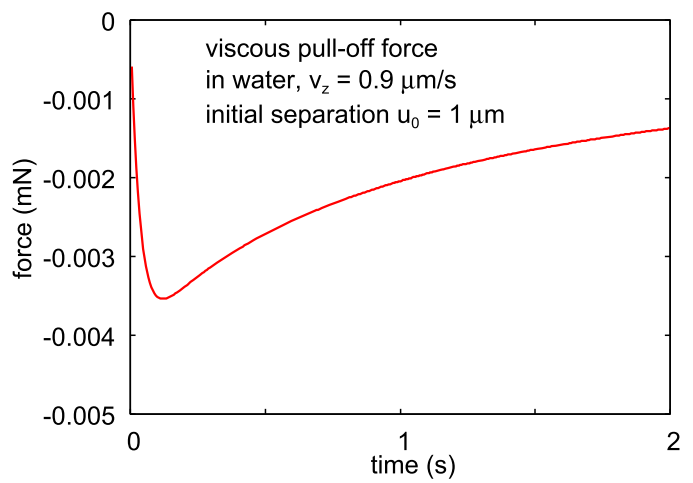

FIG. 42. The theoretically estimated interaction force between the glass ball (diameter $2 R=3 \mathrm{~cm}$ ) and a flat substrate as a function of time close to a detachment in water (viscosity $\eta=0.001 \mathrm{~Pa} \mathrm{~s}$ ). The drive speed is $v_{z}$ $=0.9 \mu \mathrm{m} / \mathrm{s}$ and the initial separation between the ball and the substrate is $u_{0}=1 \mu \mathrm{m}$. substrate during pull-off of a glass ball (diameter $2 R=3 \mathrm{~cm}$ ) in silicone oil (with the viscosity $\eta=0.35 \mathrm{~Pa} \mathrm{~s}$ ), for the pulloff speeds $v_{z}=35,6.3$, and $1.8 \mu \mathrm{m} / \mathrm{s}$. The green lines are the theoretical predictions, assuming that only a viscous drag force acts on the rubber substrate, and the different symbols are the measured data. In Fig. 23(c), we show measured data for several pull-off events, shifted along the time axis, so the minimum interaction force coincides. In the calculations, we have assumed the initial ball-substrate separation $u_{0}=1.8$, 1.65 , and $1.4 \mu \mathrm{m}$, respectively.

In Fig. 41, we show the same as in Fig. 40 but for the waiting time $7200 \mathrm{~s}$. We have assumed the initial separation (at $F=0$ ) $u_{0}=1.3 \mu \mathrm{m}$. The interfacial separations (for $F=0$ ) used above, $u_{0}=1.3-1.8 \mu \mathrm{m}$, are all consistent with what is expected from contact mechanics calculations (see Sec. III A).

For water, the fluid flow has a negligible effect on the observed pull-off force. This is illustrated in Fig. 42, which shows the theoretically estimated time dependence of interaction force between the glass ball (diameter $2 R=2.5 \mathrm{~cm}$ ) and a flat substrate in water (viscosity $\eta=0.001 \mathrm{~Pa} \mathrm{~s}$ ). The drive speed is $v_{z}=0.9 \mu \mathrm{m} / \mathrm{s}$ and the initial separation between the ball and the substrate is $u_{0}=1 \mu \mathrm{m}$. Note that the interaction force is $\sim 100$ times smaller than the measured one, and the interaction force decays slower with increasing time than observed [compare Figs. 42 and 23(b)].

${ }^{1}$ B. N. J. Persson, Sliding Friction: Physical Principles and Applications (Springer, Heidelberg, 2000).

${ }^{2}$ E. Gnecco and E. Meyer, Elements of Friction Theory and Nanotribology (Cambridge University Press, 2015).

${ }^{3}$ M. T. Siniawski, J. Felts, D. Kurilich, A. Lopez, and A. Malik, "Method for testing sliding frictional response of lubricious thin films used in plastic medical syringes," Tribol.-Mater., Surf. Interfaces 9, 113 (2015).

${ }^{4}$ W. Brostow, D. Pietkiewicz, and S. R. Wisner, "Polymer tribology in safety medical devices: Retractable syringes," Adv. Polym. Technol. 26, 56 (2007).

${ }^{5}$ C. Petersen, "Syringe siliconisation, trends, methods, analysis procedures," Int. Pharm. Ind. 4, 92 (2012).

${ }^{6}$ A. D. Roberts, "Squeeze films between rubber and glass," J. Phys. D: Appl. Phys. 4, 423 (1971)

${ }^{7}$ C. R. McClune and B. J. Briscoe, "Elastohydrodynamic films formed between a rubber cylinder and glass plate: A comparison of theory and experiment," J. Phys. D: Appl. Phys. 10, 587 (1977).

${ }^{8}$ S. C. Richards and A. D. Roberts, J. Nat. Rubber Res. 3, 210 (1988).

${ }^{9}$ A. D. Roberts and D. Tabor, Proc. R. Soc. A 325, 323 (1971); A. D. Roberts, in The Physics of Tire Friction: Theory and Experiment, edited by D. F. Hays and A. L. Browne (Plenum, New York, 1974).

${ }^{10}$ A. Koenen and A. Sanon, "Tribological and vibroacoustic behavior of a contact between rubber and glass (application to wiper blade)," Tribol. Int. 40, 1484 (2007).

${ }^{11}$ F. Deleau, D. Mazuyer, and A. Koenen, "Sliding friction at elastomer/glass contact: Influence of the wetting conditions and instability analysis," Tribol. Int. 42, 149 (2009).

${ }^{12}$ B. Lorenz, N. Rodriguez, P. Mangiagalli, and B. N. J. Persson, "Role of hydrophobicity on interfacial fluid flow: Theory and some applications," Eur. Phys. J. E 37, 57 (2015).

${ }^{13}$ B. Lorenz, B. A. Krick, N. Rodriguez, W. G. Sawyer, P. Mangiagalli, and B. N. J. Persson, "Static or breakloose friction for lubricated contacts: The role of surface roughness and dewetting," J. Phys.: Condens. Matter 25, 445013 (2013).

${ }^{14}$ B. N. J. Persson, N. Prodanov, B. A. Krick, N. Rodriguez, N. Mulakaluri, W. G. Sawyer, and P. Mangiagalli, "Elastic contact mechanics: Percolation of the contact area and fluid squeeze-out," Eur. Phys. J. E 35, 5 (2012).

${ }^{15}$ K. L. Johnson, K. Kendall, and A. D. Roberts, Proc. R. Soc. London, Ser. A 324, 301 (1971).

${ }^{16}$ M. Deruelle, H. Hervet, G. Jandeau, and L. Leger, J. Adhes. Sci. Technol. 12, 225 (1998). 
${ }^{17}$ E. Charrault, C. Gauthier, P. Marie, and R. Schirrer, "Experimental and theoretical analysis of a dynamic JKR contact," Langmuir 25, 5847 (2009).

${ }^{18}$ K. J. Wahl, S. A. S. Asif, J. A. Greenwood, and K. L. Johnson, "Oscillating adhesive contacts between micron-scale tips and compliant polymers," J. Colloid Interface Sci. 296, 178 (2006).

${ }^{19}$ L. Pastewka and M. O. Robbins, Proc. Natl. Acad. Sci. U. S. A. 111, 3298 (2014).

${ }^{20}$ B. N. J. Persson, I. M. Sivebaek, V. N. Samoilov, K. Zhao, A. I. Volokitin, and Z. Zhang, J. Phys.: Condens. Matter 20, 395006 (2008).

${ }^{21}$ G. Carbone, M. Scaraggi, and U. Tartaglino, Eur. Phys. J. E 30, 65 (2009).

${ }^{22}$ A. Chateauminois and C. Fretigny, Eur. Phys. J. E 27, 221 (2008).

${ }^{23}$ B. Z. Newby, M. K. Chaudhury, and H. R. Brown, Science 269, 1407 (1995).

${ }^{24}$ D. L. Schmidt, R. F. Brady, K. Lam, D. C. Schmidt, and M. K. Chaudhury, Langmuir 20, 2830 (2004)

${ }^{25}$ M. K. Chaudhury, Mater. Sci. Eng.: R: Rep. 16, 97 (1996).

${ }^{26}$ P. Silberzan, S. Perutz, E. J. Kramer, and M. K. Chaudhury, Langmuir 10, 2466 (1994).

${ }^{27}$ A. Ghatak, K. Vorvolakos, H. She, D. L. Malotky, and M. K. Chaudhury, J. Phys. Chem. B 104, 4018 (2000).

${ }^{28} \mathrm{~K}$. Kendall, Molecular Adhesion and its Applications: The Sticky Universe (Kluwer Academic, New York, 2001).

${ }^{29}$ B. A. Krick, J. R. Vail, B. N. J. Persson, and W. G. Sawyer, Tribol. Lett. 45, 185 (2012).

${ }^{30}$ C. Creton and M. Ciccotti, Rep. Prog. Phys. 79, 046601 (2016).

${ }^{31}$ B. N. J. Persson and E. A. Brener, Phys. Rev. E 71, 036123 (2005).

${ }^{32}$ B. N. J. Persson, O. Albohr, G. Heinrich, and H. Ueba, J. Phys.: Condens. Matter 17, R1071 (2005).

${ }^{33}$ B. N. J. Persson, Surf. Sci. Rep. 61, 201 (2006).

${ }^{34}$ B. N. J. Persson, "Adhesion between an elastic body and a randomly rough hard surface," Eur. Phys. J. E 8, 385 (2002).

${ }^{35} \mathrm{D}$. Maugis, "Adhesion of spheres: The JKR-DMT transition using a dugdale model," J. Colloid Interface Sci. 150, 243 (1992).

${ }^{36}$ S. Hyun, L. Pei, J.-F. Molinari, and M. O. Robbins, Phys. Rev. E 70, 026117 (2004).

${ }^{37}$ C. Campana and M. H. Müser, Europhys. Lett. 77, 38005 (2007).

${ }^{38}$ W. B. Dapp, A. Lucke, B. N. J. Persson, and M. H. Müser, Phys. Rev.Lett. 108, 244301 (2012).

${ }^{39}$ B. N. J. Persson, J. Chem. Phys. 115, 3840 (2001).

${ }^{40}$ S. Akarapu, T. Sharp, and M. O. Robbins, Phys. Rev. Lett. 106, 204301 (2012).

${ }^{41}$ C. Campana, B. N. J. Persson, and M. H. Müser, J. Phys.: Condens. Matter 23, 085001 (2011).

${ }^{42}$ B. N. J. Persson and M. Scaraggi, J. Chem. Phys. 141, 124701 (2014).

${ }^{43}$ B. N. J. Persson and M. Scaraggi, "Lubricated sliding dynamics: Flow factors and Stribeck curve," Eur. Phys. J. E 34, 113 (2011).

${ }^{44}$ M. Scaraggi and B. N. J. Persson, "Time-dependent fluid squeeze-out between soft elastic solids with randomly rough surfaces," Tribol. Lett. 47, 409 (2012).

${ }^{45}$ B. Lorenz, B. A. Krick, N. Mulakaluri, M. Smolyakova, S. Dieluweit, W. G. Sawyer, and B. N. J. Persson, J. Phys.: Condens. Matter 25, 225004 (2013).

${ }^{46}$ L. Dorogin, A. Tiwari, C. Rotella, P. Mangiagalli, and B. N. J. Persson, "Role of preload in adhesion of rough surfaces," Phys. Rev. Lett. 118, 238001 (2017).

${ }^{47}$ C. Y. Hui, Y. Y. Lin, and J. M. Baney, J. Polym. Sci., Part B: Polym. Phys. 38, 1485 (2000).

${ }^{48}$ B. N. J. Persson, O. Albohr, C. Creton, and V. Peveri, "Contact area between a viscoelastic solid and a hard, randomly rough, substrate," J. Chem. Phys. 120, 8779 (2004).
${ }^{49}$ A. Tiwari, L. Dorogin, A. I. Bennett, K. D. Schulze, W. G. Sawyer, M. Tahir, G. Heinrich, and B. N. J. Persson, Soft Matter 13, 3602 (2017).

${ }^{50}$ E. Kroner, R. Maboudian, and E. Arzt, Adv. Eng. Mater. 12, 398 (2010).

${ }^{51}$ E. Kroner, R. Maboudian, and E. Arzt, IOP Conf. Ser.: Mater. Sci. Eng. 5, 012004 (2009).

${ }^{52}$ E. Kroner, R. Maboudian, and E. Arzt, J. Adhes. 87, 447 (2011).

${ }^{53}$ A. Tiwari, L. Dorogin, M. Tahir, K. W. Stöckelhuber, G. Heinrich, N. Espallargas, and B. N. J. Persson, "Rubber contact mechanics: Adhesion, friction and leakage of seals," Soft Matter 13, 9103 (2017).

${ }^{54}$ See http://msdssearch.dow.com/PublishedLiteratureDOWCOM/dh_09ac/ 0901b803809ace40.pdf?filepath=consumer/pdfs/noreg/52-1247D-01.pdf\& fromPage $=$ GetDoc for the information about Dow Corning 360 Medical Fluid used in the siliconization emulsion.

${ }^{55}$ L. Mullins, Rubber Chem. Technol. 42, 339 (1969).

${ }^{56}$ A. R. Payne, "The dynamic properties of carbon black-loaded natural rubber vulcanizates. Part I,” J. Appl. Polym. Sci. 6, 57 (1962).

${ }^{57}$ See movie of evaporating water droplet at: https://www.youtube.com/ playlist?list=PLndJWxAs2yyIYJ2YB72fn9gDzgydUrfwP.

${ }^{58} \mathrm{R}$. Tadmor, "Line energy and the relation between advancing, receding, and Young contact angles," Langmuir 20, 7659 (2004).

${ }^{59}$ E. Chibowski, "Surface free energy of sulfur. Revisited I. Yellow and orange samples solidified against glass surface," J. Colloid Interface Sci. 319, 505 (2008).

${ }^{60}$ The surface energy per unit surface area for borosilica glass is about $\gamma \approx 0.3 \mathrm{~J} / \mathrm{m}^{2}$, as obtained by extrapolating the surface tension of melted glass to $T=300 \mathrm{~K}$ [see L. Shartsis and S. Spinner, J. Res. Natl. Bur. Stand. 46, 2209 (1951)]. However, glass surfaces cleaned with acetone still have some strongly bounded organic contamination (and water) which lowers the surface energy to $0.06-0.07 \mathrm{~J} / \mathrm{m}^{2}$. The organic contamination can be removed by oxygen plasma or piranha solution, but when the glass is kept in the normal atmosphere it quickly gets passivated by organic contamination. The glass surfaces in our study were cleaned only with acetone.

${ }^{61}$ See https://www.accudynetest.com/polymer_surface_data/butyl_rubber.pdf and https://www.accudynetest.com/polytable_01.htm for the information about the surface energy of bromobutyl rubber.

${ }^{62} \mathrm{~J}$. N. Israelachvili, Intermolecular and Surface Forces, 3rd ed. (Academic, London, 2011).

${ }^{63}$ P. G. de Gennes, F. Brochard-Wyart, and D. Quere, Capillary and Wetting Phenomena (Springer New York, 2004).

${ }^{64}$ J. A. Greenwood, J. Phys. D: Appl. Phys. 37, 2557 (2004).

65 J. A. Greenwood, J. Phys. D: Appl. Phys. 40, 1769 (2007).

${ }^{66}$ J. A. Greenwood, K. L. Johnson, S.-H. Choi, and M. K. Chaudhury, J. Phys. D: Appl. Phys. 42, 035301 (2008).

${ }^{67}$ D. Maugis and M. Barquins, J. Phys. D: Appl. Phys. 11, 1989 (1978).

${ }^{68}$ M. L. Williams, R. F. Landel, and D. J. Ferry, J. Am. Chem. Soc. 77, 3701 (1955).

${ }^{69}$ Y. Morishita, K. Tsunoda, and K. Urayama, Phys. Rev. E 93, 043001 (2016).

${ }^{70}$ Y. Morishita, K. Tsunoda, and K. Urayama, Polymer 108, 230-241 (2017).

${ }^{71}$ J. A. Greenwood and K. L. Johnson, Philos. Mag. 43, 697 (1981).

${ }^{72}$ E. Barthel and C. Fretigny, J. Phys. D: Appl. Phys. 42, 195302 (2009).

${ }^{73}$ L. Dorogin and B. N. J. Persson, "Contact mechanics for polydimethylsiloxane: From liquid to solid," Soft Matter 14, 1142 (2018).

${ }^{74}$ B. N. J. Persson, J. Phys.: Condens. Matter 20, 315007 (2008).

${ }^{75}$ F. R. Archibald, ASME Trans. 78, 29 (1953).

${ }^{76}$ See https://ntrs.nasa.gov/archive/nasa/casi.ntrs.nasa.gov/19730001779.pdf for the information about force/separation relation for a ball squeezed against a flat surface in a viscous fluid. 